\title{
Neptune and Triton: Essential pieces of the Solar System puzzle
}

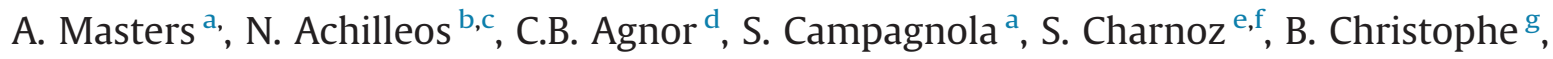

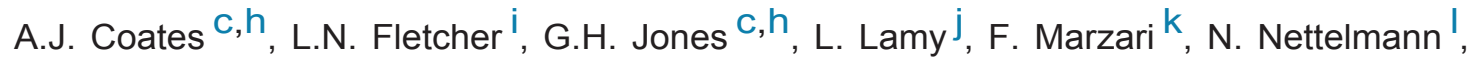

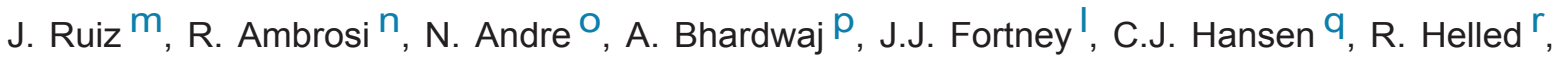 \\ G. Moragas-Klostermeyer $^{\mathrm{s}}$, G. Orton ${ }^{t}$, L. Ray ${ }^{\mathrm{b}, \mathrm{c}}$, S. Reynaud ${ }^{\mathrm{u}}, \mathrm{N}$. Sergis ${ }^{\mathrm{v}}$, R. Srama ${ }^{\mathrm{s}}$, M. Volwerk ${ }^{\mathrm{W}}$
}

\footnotetext{
a Institute of Space and Astronautical Science, Japan Aerospace Exploration Agency, 3-1-1 Yoshinodai, Chuo-ku, Sagamihara, Kanagawa 252-5210, Japan ${ }^{b}$ Atmospheric Physics Laboratory, Department of Physics and Astronomy, University College London, Gower Street, London WC1E 6BT, UK

${ }^{c}$ The Centre for Planetary Sciences at UCL/Birkbeck, Gower Street, London, WC1E 6BT, UK

d Astronomy Unit, School of Physics and Astronomy, Queen Mary University of London, Mile End Road, London, E1 4NS, UK

e Laboratoire AIM, Université Paris Diderot/CEA/CNRS, 91191 Gif-sur-Yvette Cedex, France

${ }^{\mathrm{f}}$ Institut Universitaire de France, 103 Boulevard Saint Michel, 75005 Paris, France

${ }^{g}$ ONERA - The French Aerospace Lab, 92322 Châtillon, France

h Mullard Space Science Laboratory, Department of Space and Climate Physics, University College London, Holmbury St. Mary, Dorking, Surrey RH5 6NT, UK ${ }^{i}$ Atmospheric, Oceanic \& Planetary Physics, Clarendon Laboratory, University of Oxford, Parks Road, Oxford OX1 3PU, UK

j LESIA, Observatoire de Paris, CNRS, Université Pierre et Marie Curie, Université Paris Diderot, Meudon, France

${ }^{k}$ Dipartimento di Fisica, Università di Padova, Via Marzolo 8, 35131 Padova, Italy

' Department of Astronomy and Astrophysics, University of California, Santa Cruz, CA 95064, USA

m Departamento de Geodinámica, Facultad de Ciencias Geológicas, Universidad Complutense de Madrid, 28040 Madrid, Spain

${ }^{n}$ Department of Physics and Astronomy, University of Leicester, University Road, Leicester, LE1 7RH, UK

${ }^{\circ}$ IRAP, CNRS, University Paul Sabatier Toulouse, Toulouse, France

${ }^{\mathrm{P}}$ Space Physics Laboratory, Vikram Sarabhai Space Centre, Trivandrum 695022, India

q Planetary Science Institute, Tucson, AZ 85719, USA

${ }^{r}$ Department of Geophysics and Planetary Science, Tel Aviv University, Tel Aviv 69978, Israel

s Institut für Raumfahrtsysteme, Universität Stuttgart, Pfaffenwaldring 31, 70569 Stuttgart, Germany

${ }^{t}$ MS 169-237, Jet Propulsion Laboratory, California Institute of Technology, 4800 Oak Grove Drive, Pasadena, CA 91109, USA ' Laboratoire Kastler Brossel (LKB), ENS, UPMC, CNRS, Campus Jussieu, F-75252 Paris Cedex 05, France

${ }^{v}$ Office of Space Research and Technology, Academy of Athens, Soranou Efesiou 4, 1527 Athens, Greece
}

w Space Research Institute, Austrian Academy of Sciences, Schmiedlstraße 6, 8042 Graz, Austria

The planet Neptune and its largest moon Triton hold the keys to major advances across multiple fields of Solar System science. The ice giant Neptune played a unique and important role in the process of Solar System formation, has the most meteorologically active atmosphere in the Solar System (despite its great distance from the Sun), and may be the best Solar System analogue of the dominant class of exoplanets detected to date. Neptune's moon Triton is very likely a captured Kuiper Belt object, holding the answers to questions about the icy dwarf planets that formed in the outer Solar System. Triton is geologically active, has a tenuous nitrogen atmosphere, and is predicted to have a subsurface ocean. However, our exploration of the Neptune system remains limited to a single spacecraft flyby, made by Voyager 2 in 1989. Here, we present the high-level science case for further exploration of this outermost planetary system, based on a white paper submitted to the European Space Agency (ESA) for the definition of the second and third large missions in the ESA Cosmic Vision Programme 2015-2025. We discuss all the major science themes that are relevant for further spacecraft exploration of the Neptune system, and identify key scientific questions in each area. We present an overview of the results of a European-led Neptune orbiter mission analysis. Such a mission has significant scope for international collaboration, and is essential to achieve our aim of understanding how the Solar System formed, and how it works today. 


\section{Introduction}

The primary aim of this paper is to review what we currently know about the Neptune planetary system, and to highlight the many fundamental scientific questions that remain unanswered. This review is based on a white paper that was submitted to the European Space Agency (ESA) in May 2013, to inform the selection of the science themes that will be addressed by the second and third large missions in the ESA Cosmic Vision Programme 20152025.

Neptune is classified as one of the giant planets, along with Jupiter, Saturn, and Uranus, and additionally forms a subgroup with Uranus called the "ice giants", because both planets are primarily composed of "ices" (volatile elements heavier than hydrogen and helium). However, there are fundamental and important differences between the Uranus and Neptune planetary systems, which their common classification as ice giant planets should not obscure. The Neptune system is unique, providing opportunities for major advances across multiple scientific fields that cannot be made in any other planetary environment.

Neptune orbits the Sun at a distance $\sim 30$ times greater than the mean Sun-Earth distance (an Astronomical Unit, AU). A Neptune day is just over $16 \mathrm{~h}$ long, and a planetary obliquity of $\sim 30^{\circ}$ leads to seasons over Neptune's $\sim 165$-year orbit. The planet is surrounded by a system of rings and icy moons ( 6 regular, 7 irregular). Triton, by far the largest moon, very likely formed as a dwarf planet in the Kuiper belt (like Pluto) before being captured by Neptune. This makes Triton a unique planetary satellite in the Solar System.

Voyager 2 is the only spacecraft that has encountered Neptune to date, flying by the planet on 25 August 1989 when it was summer in Neptune's southern hemisphere (Stone and Miner, 1989). Fig. 1 shows Voyager 2 imaging of Neptune during approach to the planet (Smith et al., 1989). The combination of this brief encounter and ground-based and space-based telescope observing campaigns have shown us that Neptune has the most meteorologically active atmosphere in the Solar System, despite its distance from the Sun, and that Triton has been (and could currently be) geologically active (see the review by Cruikshank (1995)). The Neptune system is barely explored compared to other planetary systems, and never with modern spacecraft instrumentation.

Sections 2 and 3 of this paper are dedicated to outlining the current state of knowledge, and defining key scientific questions, concerning the planet Neptune and its moon Triton, respectively. Each sub-section deals with one of the various science themes of Neptune/Triton science. We propose that the host of open questions put forward in Sections 2 and 3 make further spacecraft exploration of the Neptune system a priority for future Solar System exploration. Thus, in Section 4 we define further science questions that could potentially be addressed by a spacecraft bound for the outermost planet. Finally, in Section 5 we present an overview of a recent European-led Neptune orbiter mission analysis.

\section{Neptune}

\subsection{Formation and implications for the Solar System and exoplanets}

While there has been debate about Neptune's formation, a leading theory has now emerged (Gomes et al., 2005; Tsiganis et al., 2005; Morbidelli et al., 2005). It is postulated that Neptune formed at around 12-15 AU via planetesimal accumulation, before migrating to its present orbit at $\sim 30 \mathrm{AU}$ through a process of angular momentum exchange with a disk of planetesimals that initially extended out to 30-35 AU, interacting with the planets via gravitational scattering (Tsiganis et al., 2005). This scenario is

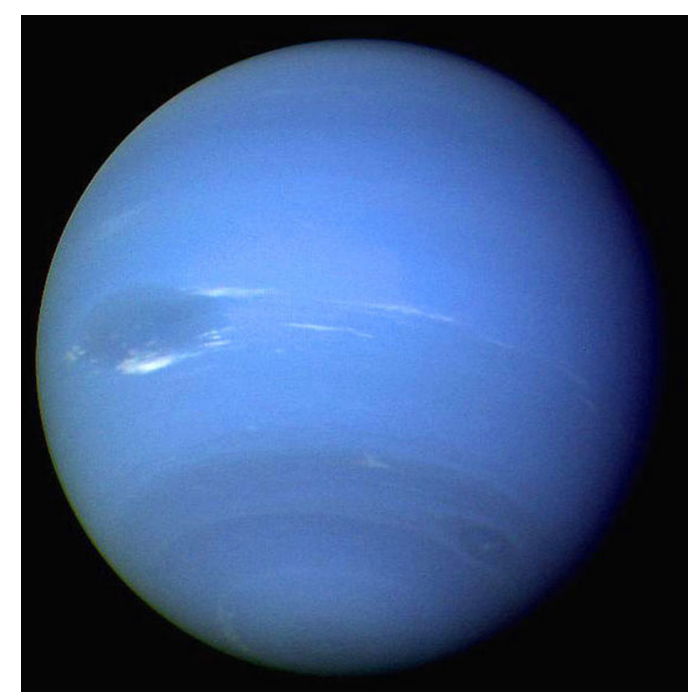

Fig. 1. Neptune, captured by the Voyager 2 narrow-angle camera. Credit: NASA/JPL.

supported by the higher density of solid material closer to the Sun (typical of protoplanetary disks) that would have lead to a shorter planetary accretion time, and explains the dynamical structure of the Kuiper Belt ( $\sim 30-50 \mathrm{AU}$, remnants of the planetesimal disk), the possible occurrence of the cataclysmic late heavy bombardment on the terrestrial planets, and the observed compositional diversity of the asteroid belt.

This leading theory highlights the importance of Neptune for Solar System formation and configuration, as illustrated in Fig. 2. Neptune effectively pushed the outer boundaries of our Solar System (Morbidelli, 2004). However, the process by which Neptune formed through accretion of planetesimals is poorly constrained. In addition, present understanding of the composition, configuration, and dynamics of the early Solar System is far from comprehensive, and our best models still cannot explain a number of features of the present day Solar System. Accurate knowledge of the physical properties of Neptune is of paramount importance for progress in all these areas. The size and mass of Neptune's core and its composition (rock/ice fraction) are crucial parameters for the improvement of planetary formation theories (Goldreich et al., 2004; Mordasini et al., 2011), and for revealing the composition of the solar nebula. Knowledge of the properties and composition of interplanetary dust at Neptune's orbit (particularly originating from comets) would also lead to significant progress in this field.

One of the mysteries concerning Neptune's formation stems from the fact that it had to form after Jupiter and Saturn, since it did not accrete as much gas as these two other giant planets. Its core likely reached completion in the later stages of solar nebula evolution, when the gas density was low due to viscous accretion and photoevaporation. How the growth and migration of Jupiter and Saturn delayed the accretion of Neptune's atmosphere is not completely clear (Jakubik et al., 2012). In this context, a detailed knowledge of the chemistry and composition of Neptune's atmosphere is essential for understanding how, where, and when the planet accreted it.

Impacts with large bodies in the early phases of their evolution have significantly affected the present state of the planets. While Uranus appears to have been radically altered by a potential giant impact (or even two) that not only tilted its spin axis (Safronov, 1966; Morbidelli et al., 2012) but also produced its low internal heat flux (Podolak and Helled, 2012), Neptune appears to have been affected by impacts in a different way. Its obliquity is comparable to that of the Earth and the interior is possibly more mixed with respect to that of Uranus, which would explain the 


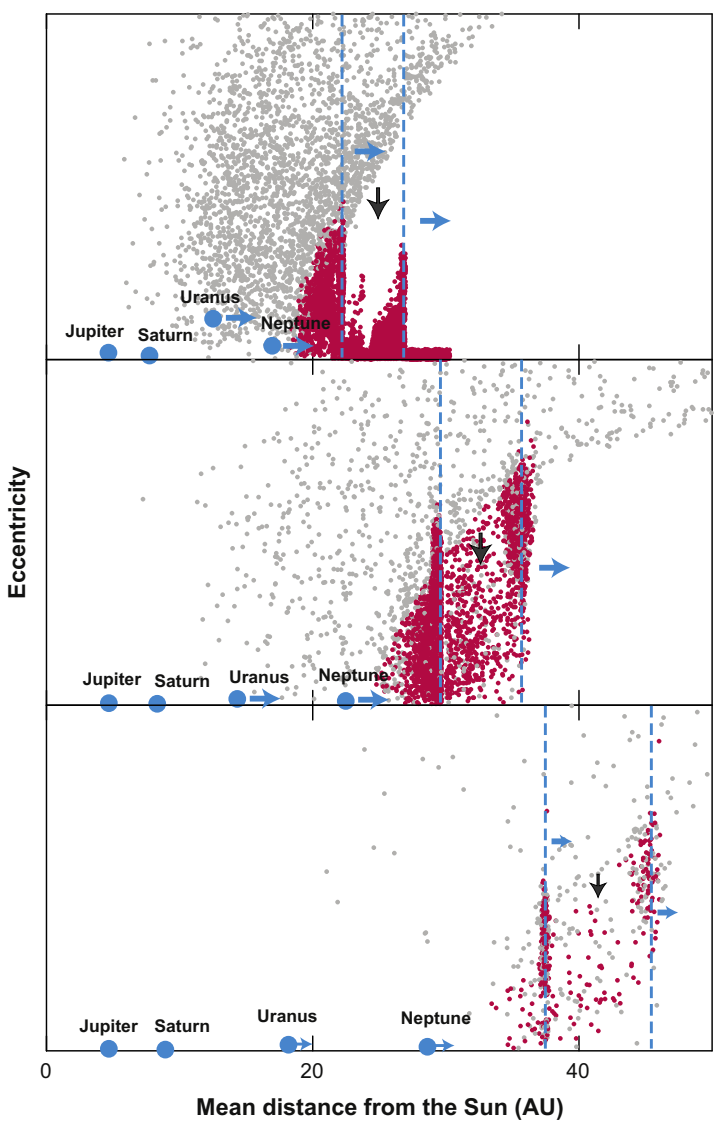

Fig. 2. The orbital evolution of the outer Solar System. The three panels show sketches of the beginning, middle, and end of planetary migration. The disk planetesimals are coloured, depending on whether they have had close encounters with Neptune (grey) or not (red). From Morbidelli (2004).

stronger internal heat source. The different collisional histories of Uranus and Neptune might also explain their different cooling histories, found to be even more prominent when using the latest atmospheric models (Fortney et al., 2011).

Focus on Neptune has intensified recently due to the discovery of numerous exoplanets with similar physical characteristics, like Gliese 436 b or GJ 3470 b. In fact, Neptune-sized and sub-Neptunesized planets are harboured by $3-31 \%$ of the Sun-like stars (Fressin et al., 2013). Neptune is expected to be typical of these similar-size exoplanets (likely more so than Uranus, which has been radically altered by collisions), and possibly shares a similar evolution. A better knowledge of Neptune's physical properties will shed new light on the formation and characteristics of these exoplanets.

Key scientific questions:

- How and where did Neptune form?

- What role did Neptune play in early Solar System dynamics?

- What does Neptune tell us about the numerous exoplanets of similar mass?

\subsection{Interior}

Although difficult to directly access through observations, the interior of Neptune holds unique information about the early Solar System and on the formation, structure, and composition of ice giant planets in general. Fortunately, some of the properties of Neptune's interior are mapped onto the observable gravity field, the magnetic field, the lower atmosphere, and the measurable luminosity, albeit not unambiguously (Hubbard et al., 1995).
Therefore, a combination of new and more accurate observations and development of consistent interior models would allow us to solve a number of major mysteries concerning planetary atmospheres, formation, and evolution. In particular, why is Neptune's measured intrinsic heat flux so high? This high intrinsic heat flux represents an important difference between Neptune and Uranus, which may be due to different formation and evolution histories of the two ice giants.

The Voyager 2 encounter with Neptune provided us with some constraints on Neptune's interior. Prior to the encounter, Neptune was thought to be layered in the form of a rocky core, surrounded by an ice shell and a hydrogen/helium envelope. Voyager data indicated a light-element component in Neptune's deep interior, and a transition from a hydrogen/helium-rich to an icy/rock-rich interior at about $60-80 \%$ of the planetary radius (Hubbard et al., 1995). Our current, still very limited, understanding of Neptune's interior is illustrated in Fig. 3.

Models constrain the light-element mass fraction in Neptune's deep interior to be $0-30 \%$ (Nettelmann et al., 2013), but this range allows for a variety of fundamentally different scenarios. For instance, a low light-element mass fraction could be explained by excess hydrogen originating from an initial water, ammonia and methane-rich composition, which was dissociated under high pressures and underwent phase separation into a hydrogenoxygen phase and a carbon-nitrogen phase. The latter phase may have produced a diamond core. In contrast, a high light-element abundance would indicate simultaneous accretion of small planetesimals and gas, as well as a rock-rich deep interior instead of an ice-rich interior.

The key questions of the abundance and metallisation of hydrogen in Neptune's deep interior, and the degree to which its interior is convective and adiabatic, are central to understanding how Neptune generates its magnetic field (see Section 2.5). Both a dynamo in a thin, ionic water shell above a stably stratified interior, and a large, metallic core dynamo have been suggested to explain the Voyager planetary magnetic field measurements. However, stable stratification over a large fraction of Neptune's interior challenges explanations for the observed high luminosity.

The fraction of heavy elements in the outer envelope of Neptune's interior is also unclear, and is related to the chemistry and composition of the tropospheric layer of Neptune's atmosphere (see Section 2.3). While adiabatic Neptune interior models allow for a reasonable deuterium enrichment in the ices, similar to that of ocean water or cometary ices (see Fig. 3a), the atmospheric oxygen abundance from adiabatic interior models does not exceed $\sim 200 \times$ protosolar, in contrast to the $\mathrm{O}: \mathrm{H}$ enrichment as inferred from atmosphere models ( $\sim 500 \times$ protosolar) that are adjusted to explain the measured tropospheric CO enrichment (Luszcz-Cook and de Pater, 2013) (see Fig. 3b).

Key scientific questions:

- Why is the heat flux from Neptune's interior so high?

- Is the magnetic field generated in a thin shell or in a metallic, convective interior?

- What is the origin and abundance of light elements in the deep interior, and of ices in the atmosphere and outer envelope?

\subsection{Atmosphere}

Despite its distance from the Sun, Neptune displays some of the most dramatic meteorological and chemical activity of any atmosphere in our Solar System, displaying zonal banding, dark ovals and sporadic clouds, along with the fastest wind speeds measured in any planetary atmosphere (up to $450 \mathrm{~m} / \mathrm{s}$, compared with 30100 m/s on Earth (Hammel et al., 1989; Ingersoll, 1990; Sromovsky 
a

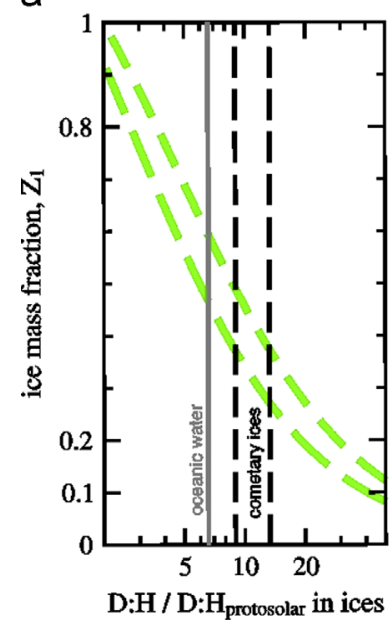

b

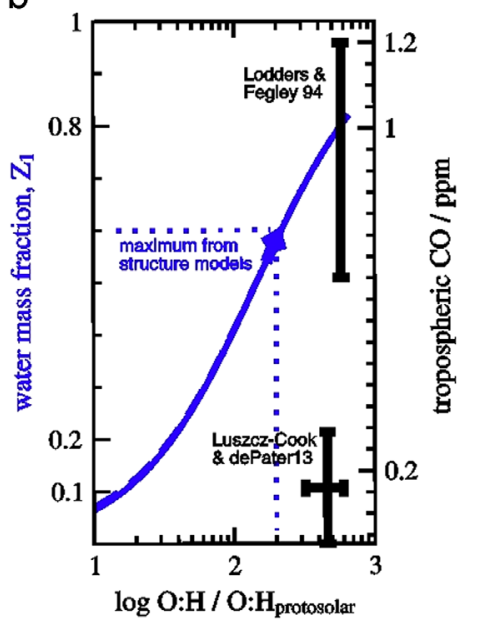

.

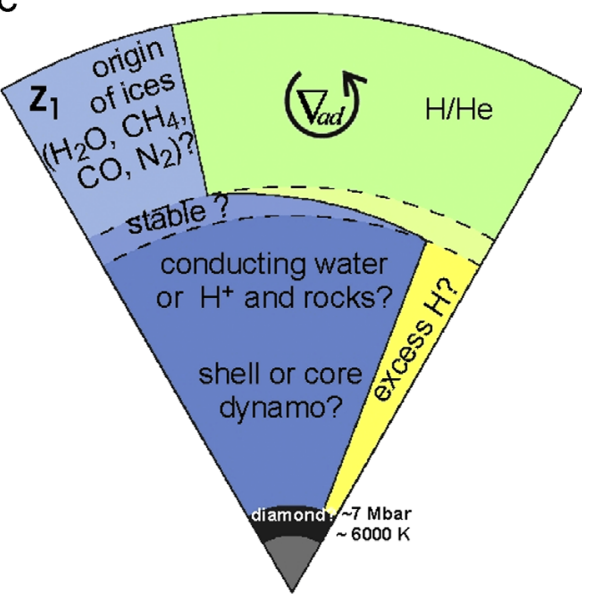

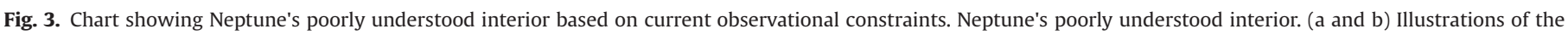

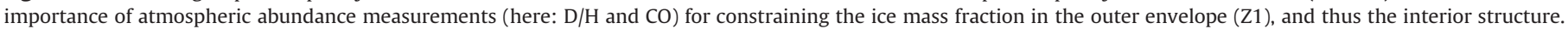
(c) Pie chart illustrating current understanding of the Neptunian interior.

et al., 2001)). Discrete cloud features, potentially comprising ices of methane and ammonia, have been observed in the visible and near-infrared, ranging from the main cloud deck at 2-3 bars up to altitudes above the tropopause at 100 mbar (e.g., Smith et al., 1989; Karkoschka, 2011; Irwin et al., 2011). Powerful zonal winds and strong latitudinal variations lead to significant shears, tearing clouds apart on timescales of hours, as shown in Fig. 4.

Given that the solar input at Neptune is only a fraction of that received by Jupiter, this strong atmospheric activity may be driven by a huge reservoir of internal heat left over from the planet's formation (see Section 2.2). Indeed, Neptune's internal heat flux produces emissions that exceed solar input by a factor of 2.6 , the largest of any planet in the Solar System (Pearl and Conrath, 1991), and in stark contrast with Uranus' apparently negligible internal heat flux.

Neptune provides an important test for models balancing seasonally dependent insolation (due to the $28^{\circ}$ axial tilt and the 165 -year orbit) and excess internal heat flux. Neptune has a different relation between banded cloud structures, atmospheric temperatures and zonal wind structure than Jupiter or Saturn. Rapidly evolving convective cloud activity seems to prevail at cool mid-latitudes (e.g., Fig. 4), and ground-based observations have shown that clouds in the main storm band at $20-40^{\circ} \mathrm{S}$ have become increasingly vigorous in the two decades since the Voyager 2 flyby (Lockwood and Jerzykiewicz, 2006; Hammel and Lockwood, 2007). Tropospheric zonal flow is characterised by strong retrograde flow at the warmer equator (e.g., Conrath et al., 1991) and a high-latitude prograde jet (e.g., Sromovsky et al., 2001) confining a seasonally variable polar vortex of unusually high temperatures and unique chemical composition (e.g., Orton et al., 2007, 2012). However, recent analysis of Neptune's gravitational field (Kaspi et al., 2013) suggests that this zonal velocity pattern is tightly confined to the outermost layers of Neptune, favouring a shallow meteorology. Dark ovals (e.g., the Great Dark Spot observed by Voyager 2 (Smith et al., 1989)) are enormous vortices, sometimes associated with bright white orographic clouds at higher altitudes. Correlating visible changes to cloud albedo, winds, eddies and vortices with environmental changes (e.g., latent heat release from cloud condensation, long-term seasonal variability in temperature and composition) are essential to understand the processes controlling the changing face of Neptune.

Some of the basic dynamical, chemical, and cloud-forming processes at work within Neptune's churning atmosphere are unknown. Neptune's atmospheric composition is determined by condensation chemistry, vertical mixing, external influx of oxygenated species from infalling comets and dust (e.g., Lellouch et al., 2010a), and a rich hydrocarbon photochemistry due to the UV destruction of methane (e.g., Orton et al., 1987; Moses et al., 2005; Greathouse et al., 2011). Knowledge of elemental enrichments $(\mathrm{C} / \mathrm{H}, \mathrm{N} / \mathrm{H}, \mathrm{O} / \mathrm{H})$, isotopic ratios $\left(\mathrm{D} / \mathrm{H},{ }^{13} \mathrm{C} /{ }^{12} \mathrm{C},{ }^{15} \mathrm{~N} /{ }^{14} \mathrm{~N}\right)$ and noble gas abundances (especially the $\mathrm{He} / \mathrm{H}_{2}$ ratio) would provide constraints on the delivery of these materials to the forming protoNeptune, and early Solar System conditions. Furthermore, mapping the spatial distributions of cloud-forming volatiles, disequilibrium species, and photochemical products would teach us about chemical processes and cloud formation at work within the ice giant, and their variability from equator to pole. The latitudinal distribution of methane (e.g., Karkoschka and Tomasko, 2011) would reveal whether it is enhanced by tropical uplift near the equator, mid-latitude convective activity, or by warming of the cold trap at the seasonally-heated poles (e.g., Orton et al., 2007). If Neptune's dynamics are analogous to those of Saturn, then its apparent polar heating would not only be the result of seasonal warming but also might contain a very compact region that is heated by a dynamically forced downdraft.

Key scientific questions:

- What drives the circulation and dynamics of the most meteorologically active atmosphere in our Solar System?

- What is the composition and structure of Neptune's atmosphere?

- What is the nature of atmospheric chemistry and cloud formation on an ice giant?

- What are the atmospheric structure and cloud properties from the troposphere to the thermosphere?

\subsection{Rings and small icy satellites}

Although all giant planets shelter a ring system, Neptune's ring system is unique because it consists of a collection of concentric and semi-transparent ringlets embedded in a tenuous sheet of dust. The Neptunian rings are tightly gravitationally coupled to a rich system of moonlets. Between the ringlets orbit a number of small moons (Naïad, Thalassa, Despina, Galatea). Both the rings and moons are especially dark, and the coupling between them is likely to be of key importance. The rings contain up to $70 \%$ dust in 


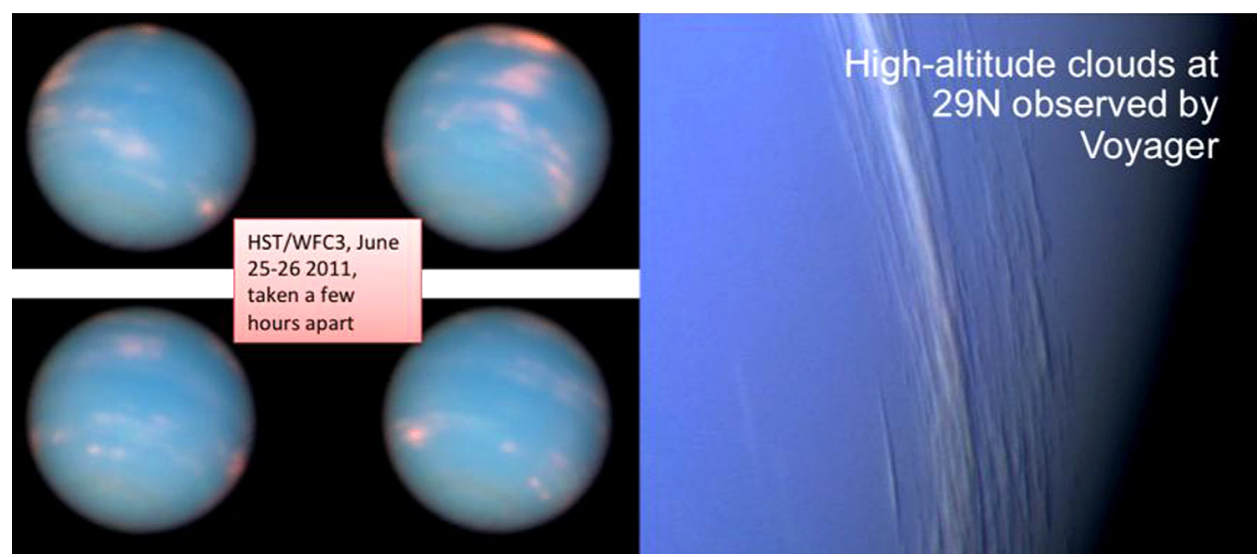

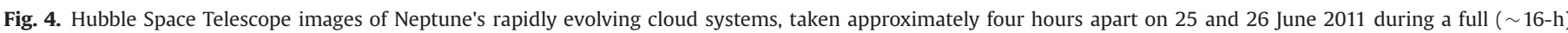
Neptune rotation (left). High-altitude clouds seen by Voyager 2 (right). Credit: NASA, ESA, and the Hubble Heritage Team (STScl/AURA).

some regions (Smith et al., 1989), which makes them fundamentally different from Saturn's rings, which contain less than $1 \%$ dust. The origin of this difference in composition is still a mystery, and could be the signature of different formation/evolutionary processes.

High-resolution imaging carried out by Voyager 2 suggests that some rings have sharp edges despite viscous spreading, suggesting gravitational confinement effects. Other rings appear to be broken into arc-like structures, as shown in Fig. 5, which are somehow able to survive despite tidal forces and collisions between ring particles. The confinement effect of one or several nearby moons has been invoked to explain this (Namouni and Porco, 2002). Earth-based observations have revealed the dynamical nature of the rings, and showed in 1999 that some arcs had shifted significantly from their expected location (Sicardy et al., 1999), while others seem to have fluctuated strongly in brightness since the Voyager era. Although the Jovian and Saturnian systems have moon-driven, extended, diffuse ring systems, currently no data exists about the Neptunian environment (Krivov et al., 2002; Srama et al., 2006).

The driver(s) of ring dynamics are unclear, and widely debated. It is thought that Neptune's rings evolve under the coupled action of sunlight, gravity, and collisional processes, but why their evolution is so different from other planetary ring systems is unknown. One of the most exciting perspectives about their origin is that they could be the result of disrupted satellites, either by tides (Leinhardt et al., 2012) or by cometary impacts (Colwell and Esposito, 1990). A re-accretion process might currently be operating.

Neptune has 6 regular moons orbiting within 5 planetary radii, forming a compact system reminiscent of Saturn's mid-sized moons. A good fraction of them seem to orbit inside Neptune's Roche limit for ice, which implies that the small moons may be denser than ice (Tiscareno et al., 2013). Tidal disruption of the weakest moons could give birth to narrow rings (Leinhardt et al., 2012). Neptune's regular satellites are barely characterised, and their mass and densities are simply inferred from modeldependent arguments concerning the evolution of the rings. The surface of Proteus, the largest of Neptune's inner satellites, appears to be densely cratered, and its non-hydrostatic shape may be the signature of past collisions, as illustrated by its large crater Pharos. The surfaces of the four innermost moons have never been imaged, representing a serious gap in our knowledge of the Neptune planetary system.

Satellite surfaces are continuously exposed to the interplanetary and interstellar meteoroid background, and ejecta from moon surfaces generates surrounding dust clouds, potentially creating ring systems (Krivov et al., 2002), and it has been proposed that

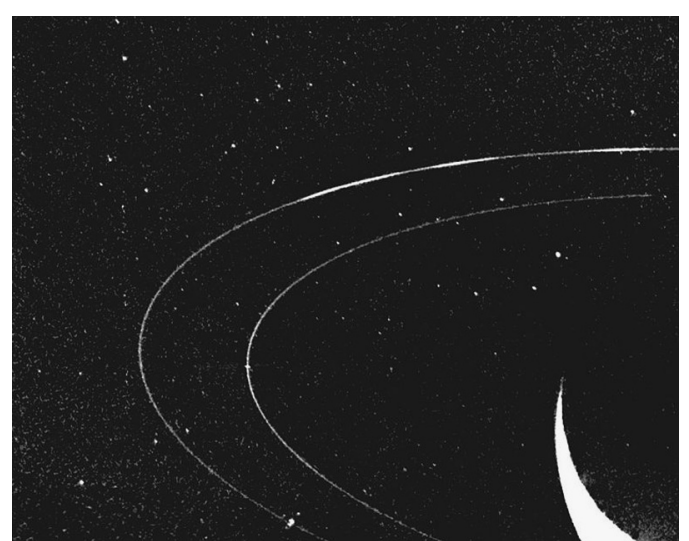

Fig. 5. Image of the Adams and Leverrier ring (outer and inner curve, respectively) taken by the Voyager 2 wide-angle camera. The brightest parts of the Adams ring are the ring arcs. Credit: NASA/JPL.

the rings might have played a role in building the satellites themselves (Crida and Charnoz, 2012). What is clear about this barely understood inner region of the Neptune system is that answering the many open questions about either the rings or inner moons would have important implications for the other.

Each of the Solar System's giant planets is known to possess distant irregular satellites on eccentric, prograde and retrograde orbits. Triton is one of these irregular satellites, and is the subject of Section 3 due its scientific importance. In addition, Neptune has the $340-\mathrm{km}$ satellite Nereid, and at least six other irregular satellites larger than about $40 \mathrm{~km}$ in size (Holman et al., 2004). These captured primitive bodies are thought to originate from the Kuiper Belt, and could provide us with important information about Neptune's history, the collisional processing of captured satellites, and the provenance and evolution of Kuiper Belt Objects (KBOs). Modelling suggests that after Triton's capture (see Section 3.1) Neptune's other irregular satellites were captured during the planet-planet encounters that occurred during the late heavy bombardment (Nesvorny et al., 2007; Vokrouhlicky et al., 2008).

Key scientific questions:

- Why is the composition of Neptune's rings different to that of any other planetary ring system, and how do the ring arcs survive?

- Does Neptune have extended, dusty rings like Jupiter and Saturn? 
- How did Neptune's inner satellites form, and how does the coupled ring-moon system work?

- Are Nereid and the other irregular satellites captured KBOs?

\subsection{Magnetic environment}

Neptune's magnetic field has a complex geometry. The single Voyager 2 flyby provided us with a limited understanding of the field structure, which nevertheless revealed a large angle of $\sim 47^{\circ}$ between the magnetic dipole and rotation axes of the planet, a dipole centre significantly offset from the centre of the planet by $\sim 0.5$ Neptune radii $\left(R_{\mathrm{N}}\right)$, and appreciable non-dipolar components (Ness et al., 1989; Connerney et al., 1991; Holme and Bloxham, 1996). The origin of such an unusual field is unclear, partly because of the lack of concrete knowledge about the planetary interior (see Section 2.2). Solving the problem of how Neptune generates its magnetic field is a major challenge for dynamo theorists, with broad implications for the field of planetary magnetism (e.g., Stanley and Bloxham, 2004; Soderlund et al., 2013).

The nature of Neptune's magnetic field leads to a highly irregular magnetosphere surrounding the planet (Bagenal, 1992). The competition between the pressure exerted by the flow of solar wind plasma from the Sun and the pressure exerted by Neptune's magnetic field produces a substantial magnetospheric cavity in the solar wind flow that envelopes most of the Neptunian satellites, including Triton. Neptune's large dipole tilt angle leads to dramatic changes in the magnetosphere in only half a planetary rotation period ( $\sim 8 \mathrm{~h}$ ), passing successively from an Earth-like to a pole-on configuration (magnetic axis parallel to the solar wind flow) every half a rotation, as illustrated in Fig. 6.

There are numerous important questions about how Neptune's magnetosphere works, which are highly relevant for understanding how it interacts with the planetary atmosphere, rings, and satellites. Uncertainty surrounds the question of how the magnetosphere changes so dramatically, and what this means for the coupling between various parts of the system. This dynamic nature makes Neptune's magnetosphere an excellent Solar System laboratory for studying charge separation and equilibration due to highly variable magnetic fields, and the timescales associated with the main regimes of plasma transport (convection, corotation) and different particle acceleration mechanisms.

The relative importance of sources and sinks of plasma in Neptune's magnetosphere is also unknown (Belcher et al., 1989; Gurnett et al., 1989; Krimigis et al., 1989; Stone et al., 1989). Triton is thought to be an important source (Richardson et al., 1991) (see Section 3), as well as charged dust particles harboured by the planet's rings. Triton makes the Neptunian magnetosphere a vital link between magnetospheres with similar internal sources of plasma but simpler internal fields (Jupiter and Saturn), and those with similar magnetic complexity but lacking such sources (Uranus). The question of whether or not there is a Triton plasma torus is an important aspect of this, and, if answered, a potentially valuable comparison could be made with moon-related plasma tori in other planetary magnetospheres. Strong dust-plasma interactions may produce charged dust streams at Neptune that are like those at Jupiter and Saturn (e.g., Kempf et al., 2005).

Auroral radio emission with a rich variety of components (smoothed, bursty) and a total radiated power of a few $10^{7} \mathrm{~W}$ has been unambiguously identified (e.g., Zarka et al., 1995). Some of this Neptune Kilometric Radiation (NKR) appears to emanate from the equatorial region (similar to Uranus' auroral radio emission), which is unique to ice giants, and makes these emissions among the most mysterious in the Solar System. The NKR provides valuable information about the plasma environment where it is produced, its modulation can shed light on the unclear dynamics at work in such an asymmetric magnetosphere, and its power is highly relevant for the poorly constrained atmospheric energy budget. In addition, $\mathrm{H} 2$ auroral emissions have been tentatively identified in the UV (Bhardwaj and Gladstone, 2000). As the furthest planet from the Sun (i.e. experiencing the lowest dynamic pressure) with a highly variable angle between the magnetic axis and the solar wind flow, how Neptune's dynamic magnetosphere interacts with the solar wind is of great interest (e.g., Schulz et al., 1995). The planetary bow shock wave that stands upstream of the magnetosphere in the solar wind flow is expected to be the strongest (highest Mach number) in the heliosphere, and the interplanetary (solar) magnetic field is very weak $(\sim 0.2 \mathrm{nT})$. As a result, the magnetopause boundary of Neptune's magnetosphere is a unique laboratory in which to study fundamental processes like magnetic reconnection, particularly in terms of plasma $\beta$ (e.g., Masters et al., 2012).

Key scientific questions:

- What is the origin and structure of Neptune's complex magnetic field?

- How does the magnetosphere re-configure on a planetary rotation timescale?

- What are the sources and sinks of magnetospheric plasma?
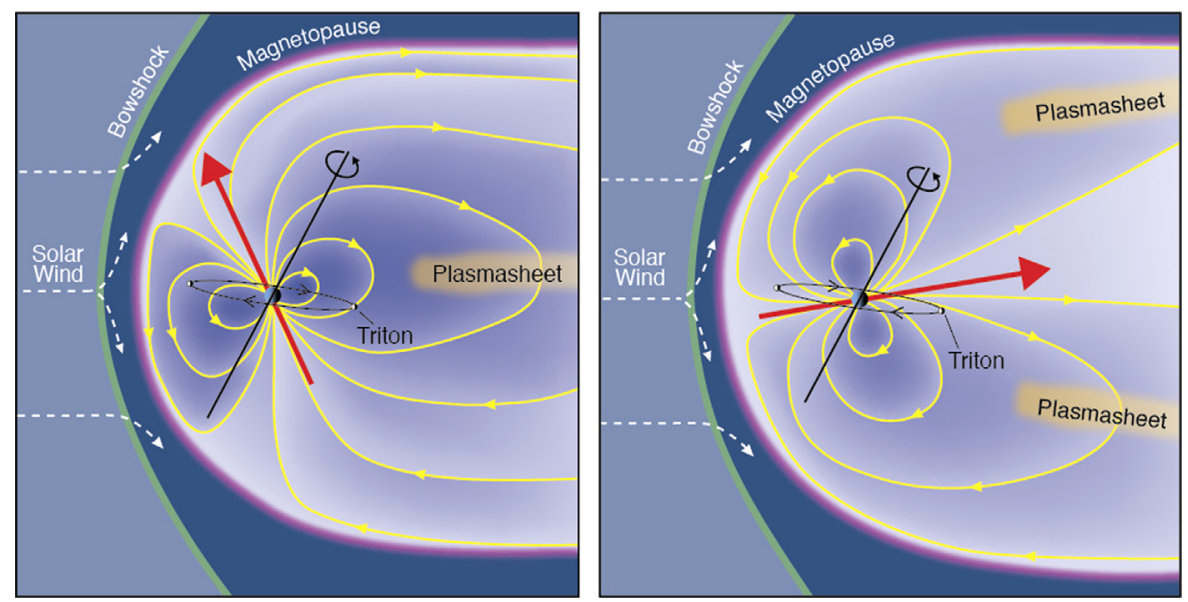

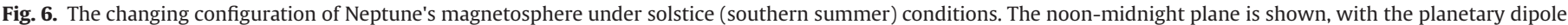
(red arrow) captured at positions separated by half a planetary rotation period. Credit: Steve Bartlett and Fran Bagenal. 
- How are Neptune's auroral emissions generated (including radio), and does this differ from the emissions observed at the Earth, Jupiter, and Saturn?

- How does Neptune's magnetosphere interact with the solar wind?

\section{Triton}

\subsection{Origin and implications for the Neptune system}

Triton, by far the largest of Neptune's moons ( $\sim 2700 \mathrm{~km}$ diameter, similar to Jupiter's moon Europa), dominates Neptune's satellite system, and is an object of tremendous scientific interest. Triton's inclined $\left(157^{\circ}\right)$ retrograde orbit strongly suggests that it was captured by Neptune at some point during its history, as illustrated in Fig. 7 (Goldreich et al., 1989; McKinnon et al., 1995; Agnor and Hamilton, 2006). Thus, Triton likely formed orbiting the Sun in a similar region as other icy dwarf planets and primitive bodies in the outer Solar System, such as Eris, Pluto, Makemake, Haumea, Sedna, Orcus, and Quaoar.

This makes Triton the only large moon in the Solar System that did not form around its host planet. The physical characteristics (e.g., composition) of Triton hold the key to understanding the icy dwarf planets of the distant Kuiper Belt, an opportunity that no other planetary system can claim. Triton is subject to the tidal, radiolytic, and collisional environment of an icy satellite, but with the initial composition of a KBO.

Triton's capture must have left it on an orbit that was much larger (orbital radius: $\sim 80-1000 R_{\mathrm{N}}$ ) and more eccentric (eccentricity: $>\sim 0.95$ ) than its current one (orbital radius: $14 R_{\mathrm{N}}$, eccentricity: 0). Triton's post-capture evolution likely dominated the subsequent evolution of the Neptunian system, and subjected the planetary satellite system to extreme processing via catastrophically disruptive collisions, gravitational scattering and tidal heating.

Driven to crossing orbits by Triton's perturbations, Neptune's inner satellites would collide at such large velocities that they would suffer catastrophic disruption and grind each other down into a debris disk (Goldreich et al., 1989). In this view, Neptune's inner satellites are either the shards left over from this process or second-generation satellites that accreted from the rings and debris disk (Crida and Charnoz, 2012) (see Section 2.4). In either case, the inner satellite system has experienced extreme collisional processing. Neptune's distant irregular satellites (exterior to Triton) were likely captured after Triton, and after the orbit of the captured Triton had circularised (Nogueira et al., 2011). These irregulars are thought to have been subsequently gravitationally

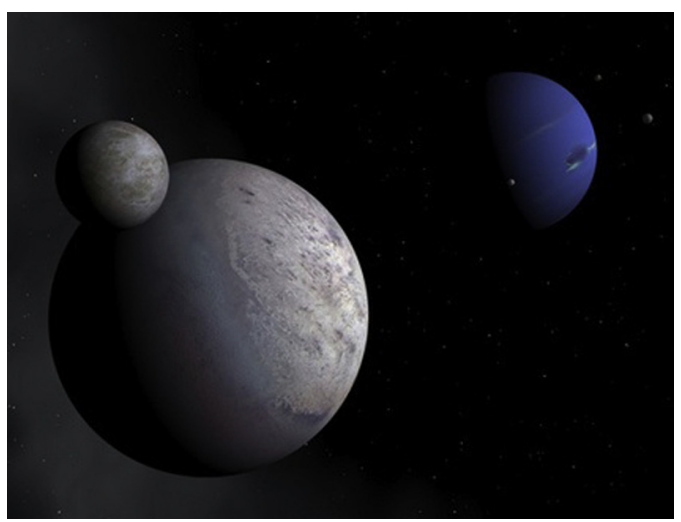

Fig. 7. Triton and a binary companion as they approached Neptune. Such an encounter may have facilitated Triton's capture by Neptune, an event that catastrophically altered the Neptune satellite system. In the image Neptune is orbited by several primordial satellites that may have existed prior to the encounter, but were destroyed in its aftermath. Credit: Craig Agnor. sculpted by the much larger moon, with satellite material being exchanged between the inner and outer regions through a variety of dynamical mechanisms.

Triton itself may have accumulated a significant portion of its mass $(\sim>20 \%)$ from the debris disk (Cuk and Gladman, 2005). The accretion of this material would have hastened Triton's orbital decay, and rendered it a composite of heliocentric and planetocentric material. Triton's orbital decay was ultimately dominated by tidal friction, and the heating during this epoch is expected to be sufficient for global melting of Triton, and the formation of subsurface oceans (McKinnon et al., 1995).

Key scientific questions:

- What physical memory does Triton retain of its heliocentric origins as an icy dwarf planet?

- How did Triton evolve after it was captured, and how did Triton affect the Neptune satellite system?

- What are the similarities and differences between Triton and the dwarf planets of the Kuiper Belt?

\subsection{Interior and surface}

The current state of our knowledge of Triton is based on very few observations (Voyager 2) and models. As a result, everything we think we know is subject to significant uncertainty, and there are fundamental questions that we have no answer to at present. What little we know includes a relatively high mean density $\left(2.065 \mathrm{~g} \mathrm{~cm}^{-3}\right)$, implying that Triton is composed of a high proportion of rock and metal ( $\sim 65-70 \%)$ compared to ice. Triton's orbital history and surface geology suggest an important role for tidal heating in the past (e.g., McKinnon et al., 1995) (see Section 3.1), which may have produced a differentiated interior with separation of ices, rocks, and metals. Triton could have a metallic core, silicate mantle, and internal liquid ocean between ice layers (Hussmann et al., 2006; McKinnon and Kirk, 2007).

Triton's surface is composed of ices, mostly $\mathrm{N}_{2}$ (which includes $\mathrm{CO}, \mathrm{CH}_{4}$, and $\mathrm{C}_{2} \mathrm{H}_{6}$ in solution), with seasonal polar deposits, plus $\mathrm{H}_{2} \mathrm{O}$, and $\mathrm{CO}_{2}$ (Quirico et al., 1999). Triton's surface has a young appearance, indicated by the sparseness and limited size of unambiguous impact craters, Crater counts indicate a surface age of several tens to hundreds of millions of years, but that in places the surface age could be as young as a few million years (Stern and McKinnon, 2000; Schenk and Zahnle, 2007). Triton's surface is therefore one of the younger surfaces in the Solar System, strongly suggesting that Triton is currently a geologically active satellite.

Triton's surface shows a variety of terrains very different to those in other icy satellites. There are two major types of geological terrains (Smith et al., 1989; Croft et al., 1995), and a large polar cap of solid nitrogen ice covers a significant fraction of the southern hemisphere. Fig. 8 shows Voyager 2 imaging of the different terrain types. A substantial portion of the surface away from the polar cap that could be imaged by Voyager 2 during its flyby appears to be occupied by expanses of regularly spaced, nearly circular depressions, dubbed cantaloupe terrains. The depressions are a few tens of kilometres wide and have a complex morphology. This kind of terrain has been interpreted to have been formed through compositional diapirism (a process caused by the gravity ascent of low-density material placed under denser layers) affecting a $\sim 20 \mathrm{~km}$ thick crustal layer (Schenk and Jackson, 1993). The other terrain type consists of undulating or smooth plains that show a variety of landforms, including terraces, and depressions filled with smooth materials and "ice lakes". The transition between both terrain types is characterised by the progressive flooding and disappearance of the cantaloupe texture, suggestive of onlapping by smooth materials emplacement. 


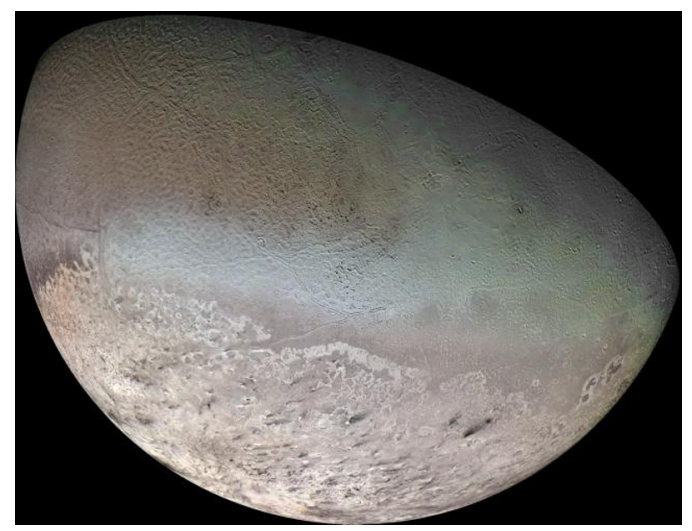

Fig. 8. Global mosaic of Triton's surface. The southern polar cap covers the lower part of the imaged region of the surface. At lower latitudes the cantaloupe terrain and plains are in the West and the East, respectively. Credit: NASA/JPL/USGS.

The surface is also deformed by a global network of ridges and troughs, more visible on the cantaloupe terrains and partly flooded at some locations on the plains (Croft et al., 1995). The ridges morphologically resemble those seen at Jupiter's moon Europa (Prockter et al., 2005), although they are much less numerous.

The brittle lithosphere (the outermost rigid layer of Triton) is estimated to be $\sim 10-15 \mathrm{~km}$ thick (Ruiz, 2003), which implies heat flows at the time when the surface was deformed that were clearly higher than those associated with the total radioactive heat production in the rocky portion of the satellite. Thus, observed resurfacing, geological activity, and the relatively thin lithosphere could have been caused by the heat generated during the capture of Triton, or by later release of the remaining heat. Indeed, tidal heating should be comparatively reduced in the current orbital eccentricity (Gaeman et al., 2012).

Voyager 2 observed at least two plumes of nitrogen gas and dust at Triton's southern polar cap, which erupted from beneath the surface, extended up to $8 \mathrm{~km}$ above it, and were then dragged by atmospheric winds (Soderblom et al., 1990) (see Section 3.3). These plumes are interpreted to be consequence of geyser-like activity, which could be powered by insulation-driven heating of the nitrogen cap (Soderblom et al., 1990). However, an endogen origin (driven by internal heat) cannot be currently discarded; this possibility would be consistent with fast ejection speed suggesting a deep source (Laufer et al., 2013). Numerous dark streaks present on the polar cap may also be a result of such plume activity.

Key scientific questions:

- What are the composition, structure, and heat flow from Triton's interior?

- What is the age of features on Triton's surface?

- How geologically active is Triton and what drives the plumes?

\subsection{Atmosphere}

Triton's tenuous atmosphere was discovered by Voyager 2, although more distant remote sensing provided indirect evidence for an atmosphere before the flyby. We know only basic properties of the atmosphere, and how Triton's atmosphere interacts with both the surface of the moon below, and Neptune's magnetosphere above, remains unclear. Yet these properties are essential for understanding energy flow though the coupled planet-moon system.

Triton's atmosphere appears to be nitrogen-rich, and sustained by ices at the surface in vapour pressure equilibrium with the atmosphere. It has been likened to the atmosphere of Pluto, although Pluto's atmosphere is also poorly understood (e.g. Olkin

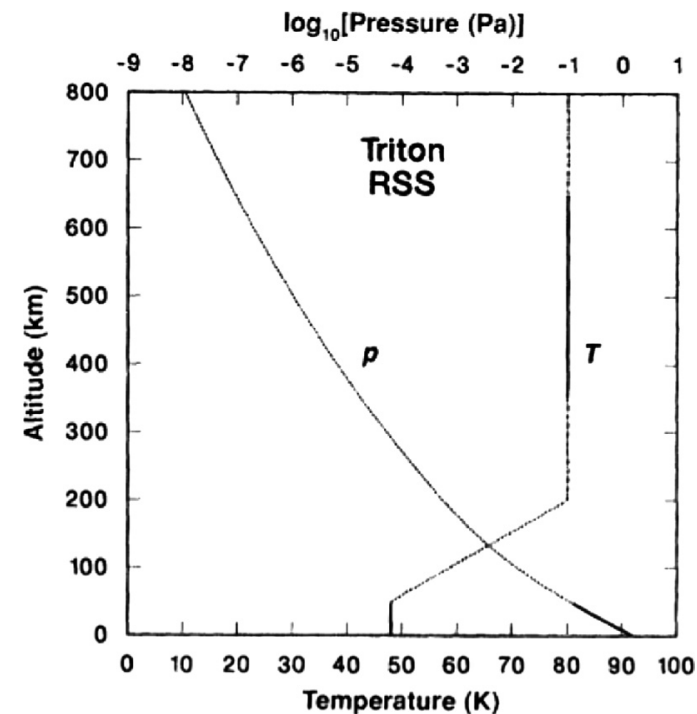

Fig. 9. Profile of Triton's atmosphere based on radio data and models. From Tyler et al. (1989).

et al., 2013). Currently known additional species in Triton's atmosphere are trace amounts of volatile gases, including methane and carbon monoxide. Trace amounts of $\mathrm{CH}_{4}$, less than those in the atmospheres of Saturn's moon Titan or Pluto, were discovered using ultraviolet observations made by Voyager (Broadfoot et al., 1989). CO was first observed using the European Southern Observatory Very Large Telescope (Lellouch et al., 2010b).

A profile of Triton's atmosphere is shown in Fig. 9. Surface atmospheric pressure is thought to be $\sim 1.4-1.9 \mathrm{~Pa}$ (14-19 $\mu \mathrm{bar}$ ) (Broadfoot et al., 1989, Tyler et al., 1989). Pressure equilibrium in the nitrogen-rich atmosphere implies an upper limit for the surface temperature of Triton of $\sim 38 \mathrm{~K}$. Triton's atmosphere is seasonally variable, as the $\mathrm{CH}_{4}$ abundance observed recently was several times that observed by Voyager (Lellouch et al., 2010b).

Turbulence at Triton's surface creates a troposphere (lower level of the atmosphere) up to $8 \mathrm{~km}$. Streaks on Triton's surface left by plumes (see Section 3.2) suggest that the troposphere is driven by seasonal winds capable of moving material over $\sim 1 \mu \mathrm{m}$ in size (Smith et al., 1989). Triton lacks a stratosphere, but has a thermosphere between $\sim 8$ and $\sim 950 \mathrm{~km}$, and an exosphere above. The temperature of the upper atmosphere is $\sim 95 \mathrm{~K}$, higher than that at the surface, which is thought to be due to heat absorbed from solar radiation and precipitation from Neptune's magnetosphere (Broadfoot et al., 1989). A haze permeates most of Triton's troposphere, which may be largely composed of hydrocarbons and nitriles created by the action of sunlight on methane. The Triton atmosphere also appears to possess clouds of condensed nitrogen that lie between 1 and $3 \mathrm{~km}$ from the surface (Smith et al., 1989).

Key scientific questions:

- What molecular species are present in Triton's atmosphere?

- What is the distribution and source of aerosols in the atmosphere?

- How do winds affect the structure of Triton's atmosphere?

- What are the properties of the nitrogen plumes?

- What is the rate of dust infall to Triton's atmosphere?

\subsection{Interaction with Neptune's magnetosphere}

Triton is thought to be the major source of plasma in Neptune's dynamic and irregular magnetosphere (Richardson et al., 1991) (see Section 2.5); however, the relative strength of Triton as a source compared to the solar wind and Neptune's ionosphere 
is unclear. Because of Triton's remarkable retrograde and highly inclined orbit, coupled with the dramatic diurnal reconfigurations of the planetary magnetosphere, the interaction between Triton and Neptune's magnetosphere is unique in the Solar System, and may be key to understanding the electrodynamics of moonmagnetosphere interactions in other planetary systems.

Triton has an ionosphere at the top of its tenuous atmosphere with a peak density at $\sim 340 \mathrm{~km}$, as determined by radio science observations. One surprise revealed by these data was the observed high ionospheric density of $\sim 46,000 \mathrm{~cm}^{-3}$ (Tyler et al., 1989); this is higher than that in the ionosphere of Saturn's moon Titan, which also has a nitrogen-based atmosphere. This is surprising because the solar illumination is a factor of $\sim 10$ lower at Triton than at Titan. The high density has been suggested to be due to the impact of energetic ( $>10 \mathrm{keV}$ ) precipitating particles from Neptune's magnetosphere (Strobel et al., 1990). The measured energy flux of $>22 \mathrm{keV}$ particles well away from Triton is $\sim 2$ orders of magnitude greater than sunlight (Krimigis et al., 1989), but this will reduce significantly when Triton is far from the planetary magnetic equator.

Due to the geometry and closest approach distance of the Voyager 2 encounter with Triton, the moon-magnetosphere interaction has never been measured directly. Triton regularly visits different regions of Neptune's magnetosphere (magnetic L-shells between 14.3 and $" 40 R_{\mathrm{N}}$ (Ness et al., 1989)) and is subject to different particle fluxes, and thus different coupling between the magnetosphere, atmosphere, and possibly Triton's surface. There is also a complex seasonal cycle, which must provide interesting and possibly significant effects.

Triton's orbital speed $\left(4.4 \mathrm{~km} \mathrm{~s}^{-1}\right)$ and the expected local speed of magnetospheric plasma flow $\left(\sim 40 \mathrm{~km} \mathrm{~s}^{-1}\right)$ mean that Triton's interaction is likely to be transonic and sub-Alfvénic (Neubauer, 1990; Strobel et al., 1990). Triton's highly conducting ionosphere affects the interaction, and magnetic field diffusion is likely to be minimal. These conditions are similar to those at Jupiter's moon Io; as a result, Alfvén wings (carrying field-aligned currents) are anticipated at Triton, as illustrated in Fig. 10. Any intrinsic (such as at Jupiter's moon Ganymede) or induced (such as at Jupiter's moon Europa) magnetic fields at Triton (e.g., due to a subsurface ocean) would clearly affect this interaction with the magnetosphere.

Key scientific objectives:

- Why is Triton's ionosphere so dense, and what production and loss processes are involved?
- What is the nature of the Triton-magnetosphere interaction, and how does it respond to constantly changing external conditions?

- How important is Triton as a source of magnetospheric plasma?

- Does Triton have an internal magnetic field or aurorae?

- To what extent do energetic particles penetrate the atmosphere?

\subsection{Habitability}

Since the era of the Voyager planetary encounters subsurface oceans have been identified at three of Jupiter's moons (Europa, Ganymede, and Callisto), and there is indirect evidence for two of Saturn's moons (Enceladus and Titan) (e.g., Kivelson, 2004). Subsurface oceans may be a common feature of icy moons in the Solar System, and a subsurface water ocean is predicted at Triton (McKinnon et al, 1995; Hussmann et al., 2006; McKinnon and Kirk, 2007). Water is thought to be a key requirement for the habitability of such an ocean. Cassini observations at Saturn's moon Enceladus have demonstrated that dust in the surrounding environment can potentially reveal the composition of any subsurface ocean (Postberg et al., 2011).

As we have seen in Section 3.2, Triton has a young surface, with active cryovolcanism likely. This is evidence for the interplay between tidal dissipation, heat transfer, and tectonics which provides the energy for resurfacing of Jupiter's satellites Europa and Ganymede and at Saturn's satellite Enceladus. Such a source of energy is another expected requirement for the habitability of a subsurface ocean. Remaining expected habitability requirements are the right chemical environment, and time. Our poor knowledge of Triton's surface and atmospheric composition are the major limitations in our assessment of Triton as a potential habitat.

As indicated above, whether a subsurface ocean exists (as predicted), and whether there is any chemical evidence for this on the surface or in the atmosphere are major open questions. Furthermore, the probable location of Triton's formation far from the locations of origin of the Jovian and Saturnian moons with subsurface oceans, and the consequent differences in composition, are highly relevant for the issue of habitability. These different origins may lead to a different chemical composition of any Triton subsurface ocean.

Key scientific questions:

- Does Triton have a subsurface ocean, and, if so, what are its properties and composition?
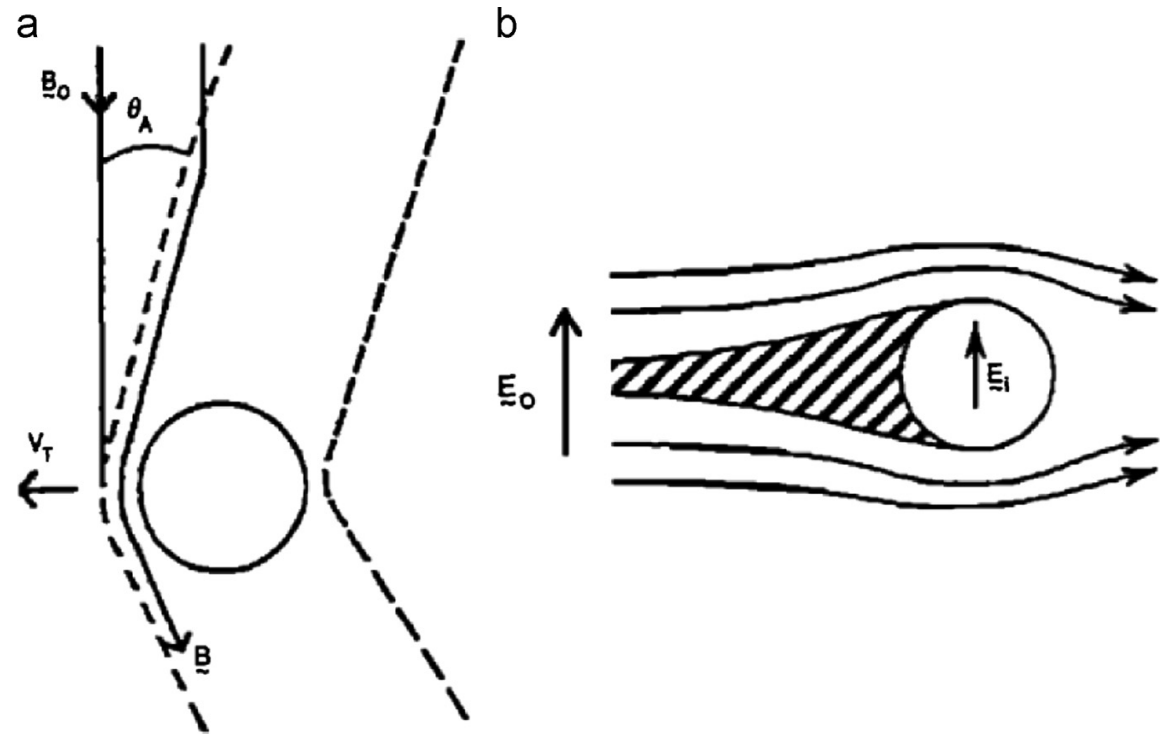

Fig. 10. Triton's magnetospheric interaction, showing the expected Alfvén wings. From Strobel et al. (1990). 
- Is the chemical environment favourable for habitability?

- How does Triton compare to other Solar System moons of astrobiological interest?

\section{Science during an interplanetary cruise to Neptune}

In Sections 2 and 3 we have presented the major themes of Neptune and Triton science, identifying key scientific questions. We propose that this host of open questions make further spacecraft exploration of the Neptune system a priority for future Solar System exploration. Motivated by this, in this section we discuss the further science themes where important open questions could potentially be addressed by a spacecraft bound for the outermost planet.

Small bodies of the outer Solar System. A spacecraft on an interplanetary cruise phase to Neptune could provide us with an excellent opportunity to characterise small bodies of the outer Solar System, with significant scientific gains. Encounters with small bodies during cruise phases have provided a wealth of data from several missions, including Galileo when travelling to Jupiter, and NEAR Shoemaker en route to its primary target Eros. Some parallels can be also be drawn with the Rosetta mission, which is dedicated to small body science.

Apart from the moons of the outer planets, no minor planetary bodies have so far been encountered beyond the asteroid belt. Between 5 and $30 \mathrm{AU}$ from the Sun, most small bodies fall into the category of Centaurs. This region is one where orbital lifetimes are typically $<10^{7}$ years (Holman and Wislom, 1993). The unstable nature of orbits in this region implies that rather than being bodies formed in situ beyond the orbit of Jupiter, most Centaurs in fact originate from the Kuiper Belt, i.e. further from the Sun, with an admixture of objects from even further afield: the Oort Cloud.

Given the expected higher volatile content of these outer Solar System small bodies compared to the typical makeup of such objects residing closer to the Sun, scientific observations in the vicinity of such bodies beyond the orbit of Jupiter would strongly complement the data gathered in situ on more volatile-poor asteroids closer to the Sun. There is a strong possibility of many of these objects being active today, such as the first Centaur found: 2060 Chiron, and 174P/Echeclus (Bauer et al. 2008). As well as the surveying of the bodies by remote sensing instruments, to characterise the surface composition and morphology, and to search for activity, particle and fields instruments should also be employed to detect the effects of any current activity on these bodies, and to search for signs of remnant magnetism.

Interplanetary and interstellar dust. Our Solar System is pervaded by dust, both interplanetary and interstellar. Continuous dust measurements in interplanetary space beyond Saturn have only been made by a few spacecraft. Modern dust detectors would provide more detailed information, together with the ability to determine the chemical composition of dust beyond Saturn's orbit for the first time, giving us essential information about the parents of the dust particles. Thus, revealing the properties of dust from 1 to $30 \mathrm{AU}$ (and particularly beyond $10 \mathrm{AU}$ ) would have implications for Solar System formation and evolution (see Section 2.1), and provide information about the Kuiper Belt. Interstellar dust grains are of particular interest as they are expected to preserve the conditions of star formation (Altobelli et al., 2003). In addition, there is potential for a comet flyby, or a crossing of a comet trail, during a cruise to the Neptune system. Dust measurements made during such encounters would also provide a link to the properties of the Oort cloud, and/or distant KBOs.

Heliospheric physics. The continuous flow of solar wind plasma away from the Sun leads to significant energy flux through our entire Solar System, and this plasma flow eventually encounters its heliopause boundary. However, very few solar wind measurements have been made in the outer Solar System, beyond $10 \mathrm{AU}$. How solar wind structures (e.g., coronal mass ejections), evolve from the Sun to Neptune is therefore a largely open question in heliospheric physics. In addition, Energetic Neutral Atoms (ENAs) have never been detected in the distant Solar System where Neptune resides. The power of ENA imaging for resolving the global structure of the heliosphere has been demonstrated by missions like IBEX and Cassini, and provides a valuable global context for in situ Voyager observations sent back from the edge of the Solar System. One of the advantages of ENA imaging at distances approaching $30 \mathrm{AU}$ is that it would allow us to simultaneously view the outer heliosphere from two different angles (when combined with Earth-based imaging), revealing the structure and dynamic behaviour of the heliosphere in more detail than has been previously possible. Another is that ENA emission is essentially computed as an line-of-sight integral that includes the ion and neutral distributions; thus, direct comparison between ENA observations made at 1 and $30 \mathrm{AU}$ could provide unique information about the physical processes operating in different regions of the Solar System.

Fundamental Physics: Testing general relativity. Interplanetary space approaching Neptune is of great importance as an environment in which we can test the limits of contemporary physics. General Relativity (GR), the current theoretical formulation of gravitation, is in good agreement with most experimental tests (Will, 2006). However, GR is a classical theory, and all attempts to merge it with the quantum description of the other fundamental interactions suggest it cannot be the final theory of gravitation. Meanwhile, experimental tests leave open windows for deviations from GR at small (Adelberger et al., 2009) and large distances (Reynaud and Jaekel, 2005)

GR is also challenged by observations at galactic and cosmic scales. The rotation curves of galaxies and the relation between redshifts and luminosities of supernovae deviate from the predictions of the theory. These anomalies are interpreted as revealing the presence of new components of the Universe, so-called "dark matter" and "dark energy" (Copeland et al., 2006; Frieman et al., 2008 ) which are thought to constitute respectively $25.8 \%$ and $69.4 \%$ of the energy content of the Universe according to most recent estimates (Ade et al., 2013). The nature of both dark matter and energy remains unknown, and, despite their contribution to total energy content, they have not been detected up to now by means other than gravitational measurements.

A crucial question when addressing the nature of dark matter and dark energy is whether or not GR is the correct description of gravity at large scales, like distances approaching that between the Sun and Neptune. Addressing this question is essential in order to bridge the gap between experiments in the Solar System and astrophysical or cosmological observations. Although past tests of general relativity with ephemerides (Fienga et al., 2010; Verma et al., 2014) and interplanetary probes (Bertotti et al., 2003) apply constraints to the nature and level of GR modifications, the accurate navigation of probes farther from the Sun is necessary to test alternative theories and effects (Hees et al., 2012). This could potentially be achieved by a spacecraft in the outer Solar Sytem equipped with appropriate accelerometer and radio science investigations (Christophe et al., 2012). Probing the limits of current gravitation theory is also closely related to the problem of Solar System formation and evolution, including the formation of the Neptune planetary system (see Section 2.1).

Key scientific questions:

- What are the characteristics of the Centaurs in the outer Solar System?

- How many of these Centaurs are active? 
- How do dust properties vary from Earth to Neptune?

- Do solar wind properties in the outer Solar System agree with model predictions?

- How do solar wind transients evolve from the Sun to $~ 30 \mathrm{AU}$, and what does this mean for Neptune's magnetospheric dynamics?

- Is general relativity the correct description of gravity at scales approaching the Sun-Neptune distance?

- If not, how does this change our understanding of Solar System formation and evolution, and the dark matter/dark energy problem?

\section{Neptune orbiter mission analysis}

As introduced in Section 1, this review of Neptune-Triton science is based on a white paper that was submitted to ESA to inform the selection of the science themes that will be addressed by the second and third large missions in the ESA Cosmic Vision Programme 2015-2025. In this section we give a brief overview of the Neptune orbiter mission concept that was presented in the white paper.

Note that the key scientific questions listed in Sections 2 and 3 are general, not related to a specific mission architecture. The key scientific questions listed in Section 4 are also somewhat general, but chosen based on the wide range of possible observations that could be made by a spacecraft bound for Neptune. The high-level mission concept presented here is an example, and can form the foundation of further analysis of an ESA-led mission to Neptune. Highlighting the identified enabling technologies is a priority. We suggest that a future mission should aim to address as many of the key scientific questions discussed in this paper as possible, through an appropriate choice of spacecraft payload.

Mission analysis heritage is provided by the most recent NASA Jet Propulsion Laboratory (JPL) mission concept study (Marley et al., 2010), the JPL-led Argo mission concept (Hansen et al., 2010a, b; Spilker et al., 2010), and the Outer Solar System Mission submitted to ESA in response to the most recent call for M-class mission proposals (Christophe et al., 2012). There is significant scope for international collaboration, and potential to use ESA JUICE mission hardware in a Neptune mission (Dougherty et al., 2011), but with far lower radiation shielding requirements.

We have identified three enabling technologies for an ESA-led Neptune orbiter mission:

1. Extended Deep Space Network (DSN) capability. Ka and X bands would be used for data and telemetry for a Neptune orbiter mission. The previous Neptune orbiter study by NASA (Marley et al., 2010) showed that a Ka-downlink to a single 34-m antenna yields 1-6 kbps at Neptune. A suggested solution to improve the data rate consisted of using four arrayed $34 \mathrm{~m}$ antennas. Although technology studies have been performed by ESOC, plans do not currently exist for multiple 35-m antennas in a single location of the European Tracking Network. However, plans exist within NASA's Deep Space Network (DSN). Use of the future DSN capability by ESA under a cooperation agreement would allow a data rate sufficient for a Neptune orbiter mission.

2. Radioisotope Thermoelectric Generators (RTGs) or Stirling Radioisotope Generators (SRGs). The issue of electrical power for any mission beyond Jupiter makes RTGs or SRGs an enabling technology for a Neptune orbiter. European RTG development activities are currently targeting a maximum electrical power output of $50 \mathrm{~W}$, with SRGs targeting $100 \mathrm{~W}$. The European programme to develop RTGs is currently at Technology Readiness Level (TRL) 3 (Ambrosi et al., 2012). The radioisotope chosen for the European space nuclear power programme is Americium-241 (Sarsfield et al., 2013; O'Brien et al. 2008) which has a longer half-life (433 years) when compared to Plutonium-238 (88 years); however, Plutonium-238 has been used in RTG systems for more than half a century. The current European RTG lifetime requirement is 20 years. Given that americium has a half-life that is much longer than any nominal mission lifetime, isotope decay will not be a limiting factor. It is also worth noting that spacecraft powered by RTG systems have exceeded their nominal mission lifetimes (e.g., Pioneer 10 exceeded its nominal 2 year mission by several decades (Dyal, 1990), and Voyager 1 is still transmitting data). Extended lifetime testing of European RTG and SRG solutions will reduce any uncertainties in lifetime values; however, this type of activity will be part of future studies. US Advanced SRGs designed for at least 17-year mission life (NASA, 2013) are currently undergoing extended lifetime testing (NASA, 2012). If we take the nominal power requirement of a Neptune orbiter mission to be $500 \mathrm{~W}, 10$ European RTGs or 5 SRGs would be sufficient, producing a total electric power of $500 \mathrm{~W}$. In the case of RTGs the mass would be of order $250 \mathrm{~kg}$, assuming a nominal specific power of $2.0 \mathrm{~W} / \mathrm{kg}$, which is the current target of a study led by a UK team (Ambrosi et al., 2012). Assuming a $20 \%$ maturity margin, the total mass would be $\sim 300 \mathrm{~kg}$. Specific power values for European SRG solutions will be determined as at the end of a current ESA study. SRG solutions for a mission to the outer planets after 2028 should not be excluded at this stage and should form part of future more detailed mission trade-off studies.

3. Solar Electric Propulsion (SEP). An RTG lifetime comparable to the interplanetary transfer time leads to a third enabling technology for a Neptune orbiter mission. Options to reduce the interplanetary transfer time are an SEP module, an Electric Sail (E-sail) (Janhunen et al., 2013), and aerocapture at Neptune Orbit Insertion (NOI). The option with the highest TRL is SEP, which would provide large Delta-V with small propellant mass in the earlier part of an interplanetary transfer to Neptune, before module ejection prior to NOI. An SEP module with four QinetiQ T6 Gridded Ion Engines (3 nominal and 1 redundant) would be sufficient, each providing $155 \mathrm{mN}$ of thrust and requiring $5.5 \mathrm{~kW}$. These high-TRL engines are flying on Alphabus, the new European GEO platform, and will also fly on BepiColombo. The power for a Neptune mission Electric Propulsion (EP) system would be provided by solar arrays (total $1 \mathrm{AU}$ power output similar to Alphabus) (specific power of $75 \mathrm{~W} / \mathrm{kg}$ at $1 \mathrm{AU}$, compared to $82 \mathrm{~W} / \mathrm{kg}$ for Dawn). An estimate of the total mass of an SEP module for a Neptune orbiter is $1500 \mathrm{~kg}$, including solar arrays, tanks, structure, and $640 \mathrm{~kg}$ of propellant. A Neptune orbiter SEP module would not be subject to degradation at high temperatures, unlike the BepiColombo SEP module.

An overview of our recent analysis of an ESA-led Neptune orbiter is given in Table 1 , and the interplanetary transfer and orbital tour are shown in Fig. 11. This is only one possible mission profile that places a spacecraft in orbit around Neptune, which makes multiple flybys of Triton. In this example, launch is in 2028 from Kourou, and the 15-year interplanetary cruise involves two Earth gravity assists and a single Jupiter gravity assist. Following NOI in 2043, the nominal orbital tour last for 2 years and includes 55 Triton flybys.

Interplanetary transfer to Neptune requires a Gravity Assist (GA) by either Jupiter or Saturn a few years after launch because of RTG lifetime and to mitigate propellant requirements. However, a Jupiter GA is more effective than a Saturn GA for a Neptune orbiter mission (Landau et al., 2009). Favourable opportunities for 
Table 1

Overview of a recent analysis of an ESA-led Neptune orbiter mission.

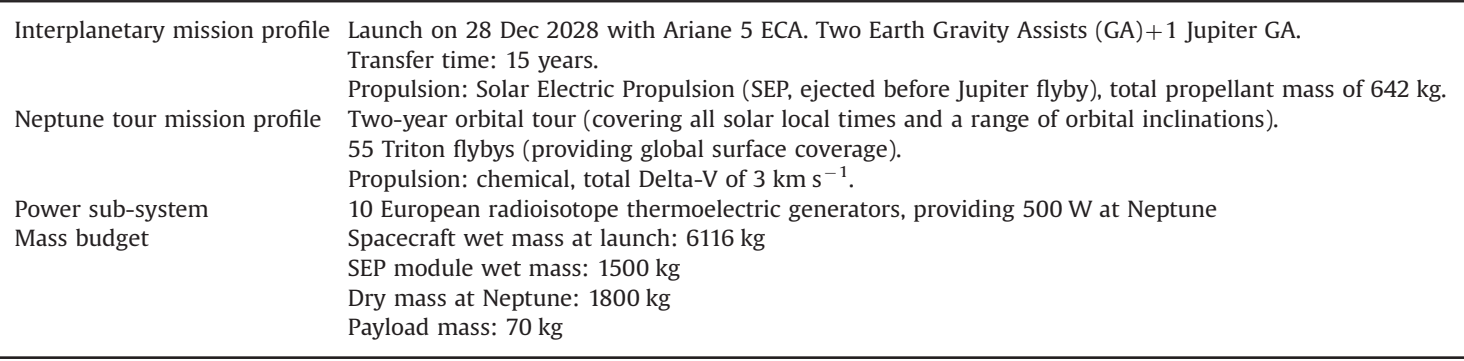
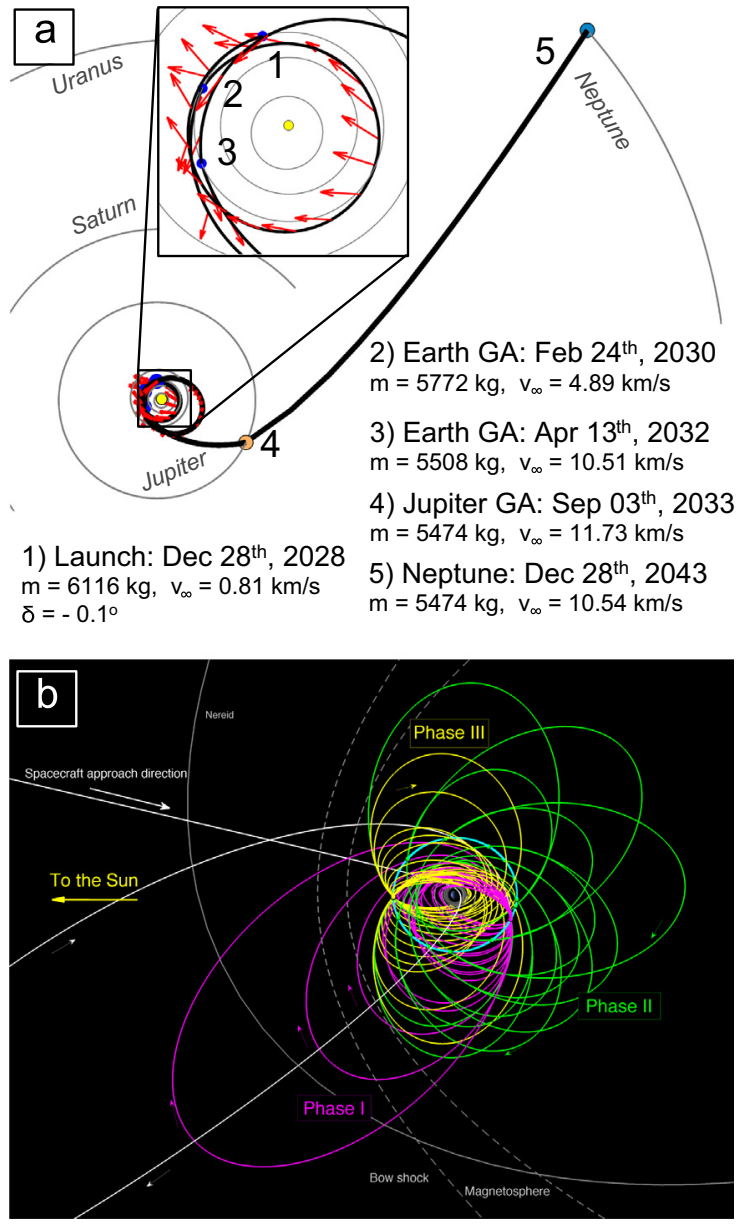

Fig. 11. Neptune orbiter mission analysis. (a) Example interplanetary transfer with launch in 2028. Trajectory arcs where solar electric propulsion is employed are represented by red arrows. (b) Example Neptune orbital tour, viewed from Neptune's north pole.

a Jupiter GA will exist in 2033 and in 2046 (separated by a JupiterNeptune synodic period of $\sim 13$ years). This example takes advantage of the 2033 Jupiter GA opportunity. A mission-enabling SEP module is employed early in the transfer (see Fig. 11), but the module is ejected prior to the Jupiter GA.

Regarding the Neptune orbital tour, we would like to highlight that Triton is an effective "tour engine", allowing a wide range of orbit trajectories and observation opportunities. Our example tour is 2 years in duration, starting with interplanetary transfer arrival conditions given by the first stage of this mission analysis. At the beginning of the tour the spacecraft flies between the inner rings and executes NOI at $3000 \mathrm{~km}$ altitude, following previous NASA mission concepts (Marley et al., 2010). During the three phases of this example tour there are inclined Neptune orbits, orbits in Triton's orbital plane, and 55 Triton flybys that cover the full range of Triton orbital locations, and altitudes between $\sim 150$ and $\sim 1000 \mathrm{~km}$. There is significant flexibility in, for example, Triton flyby altitudes, which can be raised or lowered as necessary. Our preliminary analysis suggests that a Triton orbit phase could be included at a Delta-V cost of $\sim 300 \mathrm{~m} / \mathrm{s}$, using a transfer similar to that planned for JUICE (Campagnola et al., 2012). Close flybys at Neptunian moons other than Triton are also possible.

The payload mass of $\sim 70 \mathrm{~kg}$ would be split between a number of scientific instruments. Options include (but are not limited to) a narrow-angle camera, a wide-angle camera, an infrared imager, an ultraviolet imaging spectrometer, an accelerometer, a radio science experiment (including an ultrastable oscillator), a magnetometer, a thermal imager, a range of particle detectors, a radio and plasma wave system, an ENA camera, and a dust analyser. If equipped with a payload similar to that flown on Cassini and other planetary orbiters, based on the experience of such previous missions, we expect a future Neptune orbiter to lead to paradigm-changing discoveries across the wide range of scientific themes discussed in this paper.

\section{Acknowledgements}

We are very grateful to more than 100 scientists around the world who supported the white paper on Neptune-Triton science that was submitted to ESA in May 2013 for the definition of the second and third large missions in the Cosmic Vision Programme 2015-2025. AM and SC acknowledge the support of the JAXA International Top Young Fellowship Programme. LNF was supported by a Royal Society Research Fellowship at the University of Oxford.

\section{Reference}

Ade, P.A.R., et al., 2013. Planck 2013 results. Cosmol. Parameters XVI < http://arxiv. org/abs/1303.5076>.

Adelberger, E.G., Gundlach, J.H., Heckel, B.R., Hoedl, S., Schlamminger, S., 2009. Torsion balance experiments: a low-energy frontier of particle physics. Prog. Part. Nucl. Phys, 62, 102-134.

Agnor, C.B., Hamilton, D.P., 2006. Neptune's capture of its moon Triton in a binaryplanet gravitational encounter. Nature 441, 192-194.

Altobelli, N., Kempf, S., Landgraf, M., Srama, R., Dikarev, V., Krüger, H., MoragasKlostermeyer, G., Grün, E., 2003. Cassini between Venus and Earth: Detection of interstellar dust. J. Geophys. Res. 108, A10, http://dx.doi.org/10.1029/ 2003JA009874.

Ambrosi, R.M., Williams H.R., Samara-Ratna P., Jorden, A., Slade, R., M., Jaegle, M. Koenig, J., Bannister, N.P., Deacon, T., Stuttard, M., Crawford, E.A., Vernon, D., 2012. Thermoelectric Converter System for Small-Scale RTGs, ESA TRP Report, TECS-RTG-TR-D8-001-UL.

Bagenal, F., 1992. Giant planet magnetospheres. Annu. Rev. Earth Planet. Sci. 20, 289-328.

Bauer, J.M., Choi, Y.-J., Weissman, P.R., Stansberry, J.A., Fernández, Y.R., Roe, H.G., Buratti, B.J., Sung, H.-I., 2008. The large-grained dust coma of 174P/Echeclus. Publ. Astron. Soc. Pac. 120, 393-404. 
Belcher, J.W., Bridge, H.S., Coppi, B., Gordon Jr., G.S., Lazarus, A.J., McNutt Jr., R.L., Bagenal, F., Divers, O., Eviatar, A., Ogilvie, K.W., 1989. Plasma observations near Neptune - initial results from Voyager 2. Science 246, 1478-1483.

Bertotti, B., Iess, L., Tortora, P., 2003. A test of general relativity using radio links with the Cassini spacecraft. Nature 425, 374-376.

Bhardwaj, A., Gladstone, G.R., 2000. Auroral emissions of the giant planets. Rev. Geophys. 38, 295-354.

Broadfoot, A.L., Atreya, S.K., Bertaux, J.L., Blamont, J.E., Dessler, A.J., Donahue, T.M., Forrester, W.T., Hall, D.T., Herbert, F., Holberg, J.B., Hunten, D.M., Krasnopolsky, V.A., Linick, S., Lunine, J.I., Mcconnell, J.C., Moos, H.W., Sandel, B.R., Schneider, N.M., Shemansky, D.E., Smith, G.R., Strobel, D.F., Yelle, R.V., 1989. Ultraviolet spectrometer observations of Neptune and Triton. Science 246, 1459-1466.

Campagnola, S., et al., 2012. Tisserand-leveraging transfers. Adv. Astron. Sci. 143, 1205.

Christophe, B., et al., 2012. OSS (Outer Solar System): a fundamental and planetary physics mission to Neptune, Triton and the Kuiper Belt. Exp. Astron. 34, 203.

Colwell, J.E., Esposito, L.W., 1990. A numerical model of the Uranian dust rings, Icarus 86, 530-560.

Connerney, J.E.P., Acuna, M.H., Ness, N.F., 1991. The magnetic field of Neptune. J. Geophys, Res, 96, 19023-19042.

Conrath, B.J., Flasar, F.M., Gierasch, P.J., 1991. Thermal structure and dynamics of Neptune's atmosphere from Voyager measurements. J. Geophys. Res. 96, 18931-18939.

Copeland, E.J., Sami, M., Tsujikawa, S., 2006. Dynamics of dark energy. Int. J. Mod. Phys. 15, 1753-1935.

Crida, A., Charnoz, S., 2012. Formation of regular satellites from ancient massive rings in the Solar System. Science 338, 1196.

Croft, S.K., Kargel, J.S., Kirk, R.L., Moore, J.M., Schenk, P.M., Strom, R.G., 1995. The geology of Triton. In: Cruikshank (Ed.), Neptune and Triton. University of Arizona Press, Tucson, pp. 879-947.

Cruikshank, D.P. (Ed.), 1995. Neptune and Triton. University of Arizona Press, Tucson.

Cuk, M., Gladman, B.J., 2005. Constraints on the orbital evolution of Triton. Astrophys. J. 626, L113.

Dougherty, M.K., et al., 2011. JUICE, Exploring the emergence of habitable worlds around gas giants. Assessment Study Report. European Space Agency, ESA/SRE 2011, 18 pp.

Dyal, P., 1990. Pioneers 10 and 11 deep space missions. COSPAR Colloq. Ser. 1, 373-382.

Fienga, A., Laskar, J., Kuchynka, P., Le Poncin-Lafitte, C., Manche, H., Gastineau, M., 2010. Gravity tests with INPOP planetary ephemerides. Proc. Int. Astron. Union 261, 159-169.

Fortney, J.J., Ikoma, M., Nettelmann, N., Guillot, T., Marley, M.S., 2011. Self-consistent model atmospheres and the cooling of the Solar System's giant planets. Astrophys. J. 729, 32.

Fressin, F., Torres, G., Charbonneau, D., Bryson, S.T., Christiansen, J., Dressing, C.D., Jenkins, J.M., Walkowicz, L.M., Batalha, N.M., 2013. The false positive rate of Kepler and the occurrence of planets. Astrophys. J. 766, 20.

Frieman, J.A., Turner, M.S., Huterer, D., 2008. Dark energy and the accelerating universe. Annu. Rev. Astron. Astrophys. 46, 385-432.

Gaeman, J., Hier-Majumder, S., Roberts, J.H., 2012. Sustainability of a subsurface ocean within Triton's interior. Icarus 220, 339-347.

Goldreich, P., Murray, N., Longaretti, P.Y., Banfield, D., 1989. Neptune's story. Science 245, 500-504.

Goldreich, P., Yoram, L., Re'em, S., 2004. Planet formation by coagulation: a focus on Uranus and Neptune. Annu. Rev. Astron. Astrophys. 42, 549-601.

Gomes, R., Levison, H.F., Tsiganis, K., Morbidelli, A., 2005. Origin of the cataclysmic late heavy bombardment period of the terrestrial planets. Nature 435, 466-469.

Greathouse, T.K., Richter, M., Lacy, J., Moses, J., Orton, G., Encrenaz, T., Hammel, H.B. Jae, D., 2011. A spatially resolved high spectral resolution study of Neptune's stratosphere. Icarus 214, 606-621.

Gurnett, D.A., Kurth, W.S., Poynter, R.L., Granroth, L.J., Cairns, I.H., Macek, W.M., Moses, S.L., Coroniti, F.V., Kennel, C.F., Barbosa, D.D., 1989. First plasma wave observations at Neptune. Science 246, 1494-1498.

Hammel, H.B., Beebe, R.F., de Jong, E.M., Hansen, C.J., Howell, C.D., Ingersoll, A.P., Johnson, T.V., Limaye, S.S., Magalhaes, J.A., Pollack, J.B., Sromovsky, L.A., Suomi, V.E., Swift, C.E., 1989. Neptune's wind speeds obtained by tracking clouds in Voyager images. Science 245, 1367-1369.

Hammel, H.B., Lockwood, G.W., 2007. Long-term atmospheric variability on Uranus and Neptune. Icarus 186, 291-301.

Hansen, C., et al., 2010a. Neptune Science with Argo - A Voyage through the Outer Solar System. NASA Planet. Decad. Surv., 2013-2022.

Hansen, C., et al., 2010b. Triton science with Argo - a voyage through the Outer Solar System. NASA Planet. Decad. Surv., 2013-2022.

Hees, A., Lamine, B., Reynaud, S., Jaekel, M.-T., Le Poncin-Lafitte, C., Lainey, V., Füzfa, A., Courty, J.-M., Dehant, V., Wolf, P., 2012. Radioscience simulations in general relativity and in alternative theories of gravity. Class. Quantum Grav. 29, 235027.

Holman, M.J., Wislom, J., 1993. Dynamical stability in the outer solar system and the delivery of short period comets. Astron. J. 105, 1987-1999.

Holman, M.J., Kavelaars, J.J., Grav, T., Gladman, B.J., Fraser, W.C., Milisavljevic, D., Nicholson, P.D., Burns, J.A., Carruba, V., Petit, J.-M., Rousselot, P., Mousis, O., Marsden, B.G., Jacobson, R.A., 2004. Discovery of five irregular moons of Neptune. Nature 430, 865-867.

Holme, R., Bloxham, J., 1996. The magnetic fields of Uranus and Neptune: methods and models. J. Geophys. Res. 101, 2177-2200.
Hubbard, W.B., Podolak, M., Stevenson, D.J., 1995. The interior of Neptune. In: Cruikshank (Ed.), Neptune and Triton. University of Arizona Press, Tucson, pp. $109-138$

Hussmann, H., et al., 2006. Subsurface oceans and deep interiors of medium-sized outer planet satellites and large trans-neptunian objects. Icarus 185, 258-273.

Ingersoll, A.P., 1990. Atmospheric dynamics of the outer planets. Science 248, 308-315.

Irwin, P.G.J., Teanby, N.A., Davis, G.R., Fletcher, L.N., Orton, G.S., Tice, D., Hurley, J., Calcutt, S.B., 2011. Multispectral imaging observations of Neptune's cloud structure with Gemini-North. Icarus 216, 141-158.

Jakubik, M., Morbidelli, A., Neslušan, L., Brasser, R., 2012. The accretion of Uranus and Neptune by collisions among planetary embryos in the vicinity of Jupiter and Saturn. Astron. Astrophys. 540, 16.

Janhunen, P., et al., 2013. Electric solar wind sail mass budget model. Geosci. Instrum. Methods Data Syst. 2, 85.

Karkoschka, E., 2011. Neptune's rotational period suggested by the extraordinary stability of two features. Icarus $215,439-448$.

Karkoschka, E., Tomasko, M.G., 2011. The haze and methane distributions on Neptune from HST-STIS spectroscopy. Icarus 211, 780-797.

Kaspi, Y., Showman, A.P., Hubbard, W.B., Aharonson, O., Helled, R., 2013. Atmospheric confinement of jet streams on Uranus and Neptune. Nature 497 344-347.

Kempf, S., Srama, R., Horányi, M., Burton, M., Helfert, S., Moragas-Klostermeyer, G., Roy, M., Grün, E., 2005. High-velocity streams of dust originating from Saturn. Nature 433, 289-291.

Kivelson, M.G., 2004. Moon-magnetosphere interactions: a tutorial. Adv. Space Res. 33, 2061-2077.

Krimigis, S.M., Bostrom, C.O., Cheng, A.F., Armstrong, T.P., Axford, W.I., 1989. Hot plasma and energetic particles in Neptune's magnetosphere. Science 246 1483-1489.

Krivov, A.V., Wardinski, I., Spahn, F., Krüger, H., Grün, E., 2002. Dust on the outskirts of the Jovian system. Icarus 157, 436-455.

Landau, D.F., et al., 2009. Broad search and optimization of solar electric propulsion trajectories to Uranus and Neptune. Adv. Astron. Sci. 153, 2093.

Laufer, D., Bar-Nun, A., Pat-El, I., Jacovi, R., 2013. Experimental studies of ice grain ejection by massive gas flow from ice and implications to comets, Triton and Mars. Icarus 222, 73-80.

Leinhardt, Z.M., Ogilvie, G.I., Latter, H.N., Kokubo, E., 2012. Tidal disruption of satellites and formation of narrow rings. MNRAS 424, 1419-1431.

Lellouch, E., Hartogh, P., Feuchtgruber, H., Vandenbussche, B., de Graauw, T., Moreno, R., Jarchow, C., Cavalie, T., Orton, G., Banaszkiewicz, M., Blecka, M.I., Bockelee-Morvan, D., Crovisier, J., Encrenaz, T., Fulton, T., Kuppers, M. Lara, L.M., Lis, D.C., Medvedev, A.S., Rengel, M., Sagawa, H., Swinyard, B., Szutowicz, S., Bensch, F., Bergin, E., Billebaud, F., Biver, N., Blake, G.A., Blommaert, J.A.D.L., Cernicharo, J., Courtin, R., Davis, G.R., Decin, L., Encrenaz, P. Gonzalez, A., Jehin, E., Kidger, M., Naylor, D., Portyankina, G., Schieder, R. Sidher, S., Thomas, N., de Val-Borro, M., Verdugo, E., Waelkens, C., Walker, H., Aarts, H., Comito, C., Kawamura, J.H., Maestrini, A., Peacocke, T., Teipen, R., Tils, T., Wildeman, K., 2010a. First results of Herschel-PACS observations of Neptune. Astron. Astrophys. 518, L152.

Lellouch, E., de Bergh, C., Sicardy, B., Ferron, S., Käufl, H.-U., 2010b. Detection of CO in Triton's atmosphere and the nature of surface-atmosphere interactions. Astron. Astrophys. 512, L8, http://dx.doi.org/10.1051/0004-6361/201014339.

Lockwood, G.W., Jerzykiewicz, M., 2006. Photometric variability of Uranus and Neptune, 1950-2004. Icarus 180, 442-452.

Luszcz-Cook, S.H., de Pater, I., 2013. Constraining the origins of Neptune's CO abundance with CARMA millimeter-wave observations. Icarus 222, 379-400.

Marley, M. et al., 2010. Planetary Science Decadal Survey JPL Rapid Mission Architecture Neptune-Triton KBO Study Final Report. 〈http://solarsystem. nasa.gov/2013decadal/whitepapers.cfm?Category=MS $\rangle$.

Masters, A., Eastwood, J.P., Swisdak, M., Thomsen, M.F., Russell, C.T., Sergis, N., Crary, F.J. Dougherty, M.K., Coates, A.J., Krimigis, S.M., 2012. The importance of plasma $\beta$ conditions for magnetic reconnection at Saturn's magnetopause. Geophys. Res. Lett. 39, L08103.

McKinnon, W.B., Lunine, J.I., Banfield, D., 1995. Origin and evolution of Triton. In: Cruikshank (Ed.), Neptune and Triton. University of Arizona Press, Tucson, pp. 807-877.

McKinnon, W.B., Kirk, R.L., 2007. Triton. In: Lucy-Ann McFadden, L.A., Weissman, P. Johnson, T. (Eds.), Encyclopedia of the Solar System. Academic Press, pp. 483-502.

Mordasini, C., Alibert, Y., Klahr, H., Benz, W., 2011. Theory of planet formation and comparison with observation. EPJ Web Conf. 11, 04001.

Morbidelli, A., 2004. How Neptune pushed the outer boundaries of our Solar System. Science 306, 1302-1304.

Morbidelli, A., Levison, H.F., Tsiganis, K., Gomes, R., 2005. Chaotic capture of Jupiter's Trojan asteroids in the early Solar System. Nature 435, 462-465.

Morbidelli, A., Tsiganis, K., Batygin, K., Crida, A., Gomes, R., 2012. Explaining why the uranian satellites have equatorial prograde orbits despite the large planetary obliquity. Icarus 219, 737-740.

Moses, J.I., Fouchet, T., Bezard, B., Gladstone, G.R., Lellouch, E., Feuchtgruber, H., 2005. Photochemistry and diffusion in Jupiter's stratosphere: Constraints from ISO observations and comparisons with other giant planets. J. Geophys. Res. 110, 8001

Namouni, F., Porco, C.,2002. The confinemant of Neptune's ring arcs by the moon Gelatea. Nature 417, 45-47.

Nogueira, E., Brasser, R., Gomes, R., 2011. Reassessing the origin of Triton. Icarus 214, 113-130. 
NASA Factsheets, 2013. NF-2013-07-568-HQ 〈http://solarsystem.nasa.gov/rps/ home.cfm>.

NASA, Radioisotope Power Systems Program Office, 2012. End of Fiscal Year Report $\langle$ http://rps.nasa.gov $\rangle$.

Ness, N.F., Acuna, M.H., Burlaga, L.F., Connerney, J.E.P., Lepping, R.P., 1989. Magnetic fields at Neptune. Science 246, 1473-1478.

Nesvorny, D., Vokrouhlicky, D., Morbidelli, A., 2007. Capture of irregular satellites during planetary encounters. Astron. J. 133, 1962-1976.

Nettelmann, N., Helled, R., Fortney, J.J., Redmer, R., 2013. New indication for a dichotomy in the interior structure of Uranus and Neptune from the application of modified shape and rotation data. Planet. Space Sci. 77, 143-151.

Neubauer, F.M., 1990. Satellite plasma interactions. Adv. Space Res. 10, 25-38.

O'Brien, R.C. Ambrosi, R.M., Bannister, N.P., Howe, S.D., Atkinson, H.V., 2008. Safe radioisotope thermoelectric generators and heat sources for space applications. J. Nucl. Mater. 377 (3), 506-521.

Olkin, C.B., Young, L.A., Borncamp, D., Pickles, A., Sicardy, B., Assafin, M., Bianco, F.B Buie, M.W., Dias de Oliveira, A., Gillon, M., French, R.G., Ramos Gomes Jr., A., Jehin, E., Morales, N., Opitom, C., Ortiz, J.L., Maury, A., Norbury, M., Ribas, F.B., Smith, R., Wasserman, L.H., Young, E.F., Zacharias, M., Zacharias, N., 2013. Pluto's Atmosphere Does Not Collapse 〈http://arxiv.org/abs/1309.0841〉.

Orton, G.S., Aitken, D.K., Smith, C., Roche, P.F., Caldwell, J., Snyder, R., 1987. The spectra of Uranus and Neptune at 8-14 and 17-23 $\mu \mathrm{m}$. Icarus 70, 1-12.

Orton, G.S., Encrenaz, T., Leyrat, C., Puetter, R., Friedson, A.J., 2007. Evidence for methane escape and strong seasonal and dynamical perturbations of Neptune's atmospheric temperatures. Astron. Astrophys. 473, L5-L8.

Orton, G.S., Fletcher, L.N., Liu, J., Schneider, T., Yanamandra-Fisher, P.A., de Pater, I., Edwards, M., Geballe, T.R., Hammel, H.B., Fujiyoshi, T., Encrenaz, T., Pantin, E. Mousis, O., Fuse, T., 2012. Recovery and characterization of Neptune's nearpolar stratospheric hot spot. Planet. Space Sci. 61, 161-167.

Pearl, J.C., Conrath, B.J., 1991. The albedo, eective temperature, and energy balance of Neptune, as determined from Voyager data. J. Geophys. Res. 96, 18921-18930.

Podolak, M., Helled, R., 2012. What do we really know about Uranus and Neptune? Ap. J. Lett 759, L32.

Postberg, F., Schmidt, J., Hillier, J., Kempf, S., Srama, R., 2011. A salt-water reservoir as the source of a compositionally stratified plume on Enceladus. Nature 474, 620-622.

Prockter, L.M., Nimmo, F., Pappalardo, R.T., 2005. A shear heating origin for ridges on Triton. Geophys. Res. Lett. 32, L14202, http://dx.doi.org/10.1029/ 2005 GL022832.

Quirico, E., Douté, S., Bernard, S., de Bergh, C., Cruikshank, D.P., Owen, T.C., T.R., Roush, 1999. Composition, physical state, and distribution of ices at the surface of Triton. Icarus 139, 159-178.

Reynaud, S., Jaekel, M.T., 2005. Testing the Newton law at long distances. Int. J. Mod. Phys. 20, 2294-2303.

Richardson, J.D., Belcher, J.W., Zhang, M., McNutt, R.L., 1991. Low-energy ions near Neptune. J. Geophys. Res. 96, 18993-19011.

Ruiz, J., 2003. Heat flow and depth to a possible internal ocean on Triton. Icarus 166 436-439.

Safronov, V.S., 1966. Sizes of the largest bodies falling onto the planets during their formation. Sov. Astron. 9, 987-991.

Sarsfield, M.J., et al., 2013. Progress on ${ }^{241}$ Am production for use in Radioisotope Power Systems. Proceedings of Nuclear and Emerging Technologies for Space, Albuquerque, NM, February 25-28.

Schenk, P., Jackson, P.A., 1993. Diapirism on Triton: a record of crustal layering and instability. Geology 21, 299-302.

Schenk, P.M., Zahnle, K., 2007. On the negligible surface age of Triton. Icarus 192, $135-147$.

Schulz, M., McNab, M.C., Lepping, R.P., Voigt, G.-H., 1995. Magnetospheric Configuration of Neptune. In: Cruikshank (Ed.), Neptune and Triton. University of Arizona Press, Tucson, pp. 233-277.

Sicardy, B., Roddier, F., Roddier, C., Perozzi, E., Graves, J.E., Guyon, O., Northcott, M.J., 1999. Images of Neptune's ring arcs obtained by a ground-based telescope. Nature 400, 731-733.
Smith, B.A., Soderblom, L.A., Banfield, D., Barnet, C., Basilevsky, A.T., Beebe, R.F., Bollinger, K., Boyce, J.M., Brahic, A., Briggs, G.A., Brown, R.H., Chyba, C., Collins, S.A., Colvin, T., Cook II, A.F., Crisp, D., Croft, S.K., Cruikshank, D., Cuzzi, J.N., Danielson, G.E., Davies, M.E., De Jong, E., Dones, L., Godfrey, D., Goguen, J., Grenier, I., Haemmerle, V.R., Hammel, H., Hansen, C.J., Helfenstein, C.P., Howell, C., Hunt, G.E., Ingersoll, A.P., Johnson, T.V., Kargel, J., Kirk, R., Kuehn, D.I., Limaye, S., Masursky, H., McEwen, A., Morrison, D., Owen, T., Owen, W., Pollack, J.B., Porco, C.C., Rages, K., Rogers, P., Rudy, D., Sagan, C., Schwartz, J., Shoemaker, E.M., Showalter, M., Sicardy, B., Simonelli, D., Spencer, J., Sromovsky, L.A., Stoker, C., Strom, R.G., Suomi, V.E., Synott, S.P., Terrile, R.J., Thomas, P., Thompson, W.R., Verbiscer, A., Veverka, J., 1989. Voyager 2 at Neptune - imaging science results. Science 246, 1422-1449.

Soderblom, L.A., Kieffer, S.W., Becker, T.L., Brown, R.H., Cook II, A.F., Hansen, C.J., Johnson, T.V., Kirk, R.L., Shoemaker, E.M., 1990. Triton's geyser-like plumes: discovery and basic characterization. Science 250, 410-415.

Soderlund, K.M., Heimpel, M.H., King, E.M., Aurnou, J.M., 2013. Turbulent models of ice giant internal dynamics: dynamos, heat transfer, and zonal flows. Icarus 224, 97-113.

Spilker, L.J., et al., 2010. Neptune ring science with Argo - a voyage through the Outer Solar System. NASA Planet. Decad. Surv., 2013-2022.

Srama, R., Kempf, S., Moragas-Klostermeyer, G., Helfert, S., Ahrens, T.J., Altobelli, N., Auer, S., Beckmann, U., Bradley, J.G., Burton, M., Dikarev, V.V., Economou, T., Fechtig, H., Green, S.F., Grande, M., Havnes, O., Hillier, J.K., Horanyi, M., Igenbergs, E., Jessberger, E.K., Johnson, T.V., Krüger, H., Matt, G., McBride, N., Mocker, A., Lamy, P., Linkert, D., Linkert, G., Lura, F., McDonnell, J.A.M., Möhlmann, D., Morfill, G.E., Postberg, F., Roy, M., Schwehm, G.H., Spahn, F., Svestka, J., Tschernjawski, V., Tuzzolino, A.J., Wäsch, R., Grün, E., 2006. in situ dust measurements in the inner Saturnian system. Planet. Space Sci. 54, 967-987.

Sromovsky, L.A., Fry, P.M., Dowling, T.E., Baines, K.H., Limaye, S.S., 2001. Coordinated 1996 HST and IRTF imaging of Neptune and Triton. III. Neptune's atmospheric circulation and cloud structure. Icarus $149,459-488$.

Stanley, S., Bloxham, J., 2004. Convective-region geometry as the cause of Uranus' and Neptune's unusual magnetic fields. Nature 428 (151), 153.

Stern, S.A., McKinnon, W.B., 2000. Triton's surface age and impactor population revisited in light of Kuiper belt fluxes: evidence for small Kuiper belt objects and recent geological activity. Astron. J. 119, 945-952.

Stone, E.C., Miner, E.D., 1989. The Voyager 2 encounter with the Neptunian system. Science 246, 1417-1421.

Stone, E.C., Cummings, A.C., Looper, M.D., Selesnick, R.S., Lal, N., McDonald, F.B., Trainor, J.H., 1989. Energetic charged particles in the magnetosphere of Neptune. Science 246, 1489-1494.

Strobel, D.F., Cheng, A.F., Summers, M.E., Strickland, D.J., 1990. Magnetospheric interaction with Triton's ionosphere. Geophys. Res. Lett. 17, 1661-1664.

Tiscareno, M.S., Hedman, M.M., Burns, J.A., Castillo-Rogez, J., 2013. Compositions and origins of outer planet systems: insights from the Roche critical density. Astrophys. J. Lett. 765, 5.

Tsiganis, K., Gomes, R., Morbidelli, A., Levison, H.F., 2005. Origin of the orbital architecture of the giant planets of the Solar System. Nature 435, 459-461.

Tyler, G.L., Sweetnam, D.N., Anderson, J.D., Borutzki, S.E., Campbell, J.K., Kursinski, E. R., Levy, G.S., Lindal, G.F., Lyons, J.R., Wood, G.E., 1989. Voyager radio science observations of Neptune and Triton. Science 246, 1466-1473.

Verma, A.K., Fienga, A., Laskar, J., Manche, H., Gastineau, M., 2014. Use of MESSENGER radioscience data to improve planetary ephemeris and to test general relativity. Astron. Astrophys. 561, 13.

Vokrouhlicky, D., Nesvorny, D., Levison, H.F., 2008. Irregular satellite capture by exchange reactions. Astron. J. 136, 1463-1476.

Will, C.M., 2006. The confrontation between general relativity and experiment. Living Rev. Relat. 9, 1.

Zarka, P., Pedersen, B.M., Lecacheux, A., 1995. Radio emission from Neptune. In: Cruikshank (Ed.), Neptune and Triton. University of Arizona Press, Tucson, pp. 341-387. 